\title{
Numerical Investigation of Subharmonic Solutions to Duffing's Equation
}

\author{
By \\ Minoru URABE
}

\section{Introduction}

The present paper is concerned with subharmonic solutions to Duffing's equation

$$
\frac{d^{2} q}{d \tau^{2}}+\alpha \frac{d q}{d \tau}+\kappa^{2} q\left(1+\beta q^{2}\right)=P \cos \gamma \tau .
$$

As far as the author is aware, the analytical or experimental investigation of subharmonic solutions to Duffing's equation (for analytical investigation, e.g. see [6], [10], [11], [12] and, for experimental investigation, e.g. see [5]) has been limited till recently to the equation in which the nonlinear term is small, that is, $|\beta| \ll 1$. Recently, for the strongly nonlinear equation, that is, the equation in which the nonlinear term is not necessarily small, subharmonic solutions have been investigated analytically by P. A. T. Christopher $[2,3,4]$ by the use of the method developed by Cesari [1], and numerically by $M$. E. Levenson $[7,8]$ by the use of a digital computer and by C.A. Ludeke and J.E. Cornett [9] by the use of an analog computer. Christopher established analytically the existence of a subharmonic solution of order one-third in some region of parameters, but the region of parameters obtained by him does not seem to be large enough for practical use. Numerical investigations by Levenson, Ludeke and Cornett are all based on step-by-step numerical integration of ordinary differential equations and they do 
not provide the mathematical guarantee for the existence of a subharmonic solution.

In his papers $[13,16]$, for nonlinear periodic differential systems, the author established a mathematical theory of Galerkin's procedure and gave a practical method of getting an error bound to a periodic approximate solution obtained by Galerkin's procedure. In his method, in the course of calculation of an error bound, the existence of an exact periodic solution can be assured automatically and moreover the stability of a periodic solution can be decided easily. In the present paper, making use of the above method, we have computed approximations to subharmonic solutions of order one-third for various values of parameters and calculated error bounds to the approximations. Naturally the existence of the corresponding subharmonic solutions has been assured and, in addition, the stability of these subharmonic solutions has been decided. In the present paper, harmonic solutions related with subharmonic solutions has been also computed.

By the transformation

$$
\kappa \tau=t, \quad \frac{\kappa^{2}}{P} q=x, \frac{\alpha}{\kappa}=\sigma, \frac{\beta P^{2}}{\kappa^{4}}=\varepsilon, \quad \frac{\gamma}{\kappa}=\omega,
$$

equation (1.1) can be reduced to the equation

$$
\frac{d^{2} x}{d t^{2}}+\sigma \frac{d x}{d t}+x\left(1+\varepsilon x^{2}\right)=\cos \omega t
$$

which, replacing $\omega t$ by $t$, one can rewrite as follows :

$$
\frac{d^{2} x}{d t^{2}}+\frac{\sigma}{\omega} \frac{d x}{d t}+\frac{1}{\Omega} x\left(1+\varepsilon x^{2}\right)=\frac{1}{\Omega} \cos t
$$

where

$$
\Omega=\omega^{2} \text {. }
$$

To a subharmonic solution of order one-third to (1.1), corresponds a solution to $(1.3)$ of the form

$$
x(t)=c_{1}+\sum_{n=1}^{\infty}\left(c_{2 n} \sin \frac{n}{3} t+c_{2 n+1} \cos \frac{n}{3} t\right) .
$$


Hence replacing $t$ by $3 t$ in (1.3) and (1.5), one can reduce the problem to the one to find a solution of the form

$$
x(t)=c_{1}+\sum_{n=1}^{\infty}\left(c_{2 n} \sin n t+c_{2 n+1} \cos n t\right)
$$

to the equation

$$
\frac{d^{2} x}{d t^{2}}+\frac{3 \sigma}{\omega} \frac{d x}{d t}+\frac{9}{\Omega} x\left(1+\varepsilon x^{2}\right)=\frac{9}{\Omega} \cos 3 t .
$$

In the present paper, we assume that

$$
\varepsilon>0 \text {. }
$$

For equation (1.7) with $\sigma=0$ (that is, the equation with damping absent), from the symmetricity of the equation, we have sought solutions of the form

$$
x(t)=\sum_{n=1}^{\infty} a_{n} \cos (2 n-1) t .
$$

For equation (1.7) with $\sigma \neq 0$ (that is, the equation with damping present), we have sought solutions of the general form (1.6) for small $\sigma>0$.

The computations in the present paper have been carried out by the use of TOSBAC 3400 at Research Institute for Mathematical Sciences, Kyoto University. The author wishes to acknowledge the assistance of Mrs. S. Asako, who has written the programs for TOSBAC 3400 .

\section{Galerkin's Procedure}

2. 1 Galerkin's procedure. Consider a real periodic differential system

$$
\frac{d \boldsymbol{x}}{d t}=\boldsymbol{X}(\boldsymbol{x}, t),
$$

where $\boldsymbol{x}$ and $\boldsymbol{X}(\boldsymbol{x}, t)$ are vectors of the same dimension and $\boldsymbol{X}(\boldsymbol{x}, t)$ is periodic in $t$ of period $2 \pi$. To get an approximation to a $2 \pi-$ periodic solution to $(2.1)$, we consider a trigonometric polynomial

$$
\boldsymbol{x}_{m}(t)=\boldsymbol{c}_{1}+\sum_{n=1}^{m}\left(\boldsymbol{c}_{2 n} \sin n t+\boldsymbol{c}_{2 n+1} \cos n t\right)
$$


with unknown coefficients $\boldsymbol{c}_{1}, \boldsymbol{c}_{2}, \boldsymbol{c}_{3}, \cdots, \boldsymbol{c}_{2 m}, \boldsymbol{c}_{2 m+1}$, and we determine these unknown coefficients by the equation

$$
\begin{aligned}
\frac{d \boldsymbol{x}_{m}(t)}{d t}= & \frac{1}{2 \pi} \int_{0}^{2 \pi} \boldsymbol{X}\left[\boldsymbol{x}_{m}(s), s\right] d s \\
& +\frac{1}{\pi} \sum_{n=1}^{m}\left\{\sin n t \cdot \int_{0}^{2 \pi} \boldsymbol{X}\left[\boldsymbol{x}_{m}(s), s\right] \sin n s \cdot d s\right. \\
& \left.+\cos n t \cdot \int_{0}^{2 \pi} \boldsymbol{X}\left[\boldsymbol{x}_{m}(s), s\right] \cos n s \cdot d s\right\} .
\end{aligned}
$$

Equation (2.3) is evidently equivalent to the equation

$$
\left\{\begin{array}{l}
\boldsymbol{F}_{1}(\boldsymbol{c}) \triangleq \frac{1}{2 \pi} \int_{0}^{2 \pi} \boldsymbol{X}\left[\boldsymbol{x}_{m}(t), t\right] d t=0, \\
\boldsymbol{F}_{2 n}(\boldsymbol{c}) \triangleq \frac{1}{\pi} \int_{0}^{2 \pi} \boldsymbol{X}\left[\boldsymbol{x}_{m}(t), t\right] \sin n t \cdot d t+n \boldsymbol{c}_{2 n+1}=0, \\
\boldsymbol{F}_{2 n+1}(\boldsymbol{c}) \triangleq \frac{1}{\pi} \int_{0}^{2 \pi} \boldsymbol{X}\left[\boldsymbol{x}_{m}(t), t\right] \cos n t \cdot d t-n c_{2 n}=0 \\
(n=1,2, \cdots, m),
\end{array}\right.
$$

where $\boldsymbol{c}=\operatorname{col}\left(\boldsymbol{c}_{1}, \boldsymbol{c}_{2}, \boldsymbol{c}_{3}, \cdots, \boldsymbol{c}_{2 m}, \boldsymbol{c}_{2 m+1}\right) . \quad$ A trigonometric polynomial with coefficients satisfying (2.4) will be called a Galerkin approximation of order $m$ to a $2 \pi$-periodic solution to (2.1) and the equation (2.4) will be called a determining equation for Galerkin approximations of order $m$. A method of getting an approximation to a $2 \pi$-periodic solution by computing a Galerkin approximation is called a Galerkin's procedure.

Galerkin's procedure can be justified mathematically by the following theorem.

Theorem 1. Suppose that $\boldsymbol{X}(\boldsymbol{x}, t)$ and its Jacobian matrix $\Psi(\boldsymbol{x}, t)$ with respect to $\boldsymbol{x}$ are continuously differentiable with respect to $\boldsymbol{x}$ and $t$ in the region $D \times L$, where $D$ is a closed bounded region of the $\boldsymbol{x}$-space and $L$ is the real line. If differential system (2.1) possesses an isolated $2 \pi$-periodic solution $\boldsymbol{x}=\hat{\boldsymbol{x}}(t)$ lying inside $D$, then for sufficiently large $m_{0}$, there is a Galerkin approximation $\boldsymbol{x}=\overline{\boldsymbol{x}}_{m}(t)$ to any order $m \geqq m_{0}$ such that

$$
\overline{\boldsymbol{x}}_{m}(t) \rightarrow \hat{\boldsymbol{x}}(t), \quad \dot{\overline{\boldsymbol{x}}}_{m}(t) \rightarrow \dot{\hat{\boldsymbol{x}}}(t) \quad(\cdot=d / d t)
$$

uniformly as $m \rightarrow \infty$. 
For the proof of the theorem, see [13].

By an isolated $2 \pi$-periodic solution, is meant a $2 \pi$-periodic solution such that the multipliers of solutions of the relative first variation equation are all different from unity.

2. 2 Determining equation for Duffing's equation with damping present. Clearly equaton (1.7) is of the form

$$
\ddot{x}=X(x, \dot{x}, t) \quad(\cdot=d / d t),
$$

where $X(x, y, t)$ is periodic in $t$ of period $2 \pi$. Equation (2.5) is clearly equivalent to the first order system

$$
\left\{\begin{array}{l}
\dot{x}=y \\
\dot{y}=X(x, y, t) .
\end{array}\right.
$$

For (2.6), a Galerkin approximation of order $m$ is of the form

$$
\left\{\begin{array}{l}
x_{m}(t)=c_{1}+\sum_{n=1}^{m}\left(c_{2 n} \sin n t+c_{2 n+1} \cos n t\right), \\
y_{m}(t)=\sum_{n=1}^{m}\left(-n c_{2 n+1} \sin n t+n c_{2 n} \cos n t\right) .
\end{array}\right.
$$

Hence, for (2.6), the determining equation for Galerkin approximations of order $m$ can be reduced to the equation [16]

$$
\left\{\begin{array}{c}
F_{1}(\boldsymbol{c}) \triangleq \frac{1}{2 \pi} \int_{0}^{2 \pi} X\left[x_{m}(t), y_{m}(t), t\right] d t=0, \\
F_{2 n}(\boldsymbol{c}) \triangleq \frac{1}{\pi} \int_{0}^{2 \pi} X\left[x_{m}(t), y_{m}(t), t\right] \sin n t d t+n^{2} c_{2 n}=0, \\
F_{2 n+1}(\boldsymbol{c}) \triangleq \frac{1}{\pi} \int_{0}^{2 \pi} X\left[x_{m}(t), y_{m}(t), t\right] \cos n t d t+n^{2} c_{2 n+1}=0 \\
(n=1,2, \cdots, m),
\end{array}\right.
$$

where $\boldsymbol{c}=\operatorname{col}\left(c_{1}, c_{2}, c_{3}, \cdots, c_{2 m}, c_{2 m+1}\right)$. Equation (2.8) will be called a determining equation for Galerkin approximations for the second order equation (2.5).

2. 3 Determining equation for Duffing's equation and damping absent. As is seen from (1.7), Duffing's equation with damping absent is of the form

$$
\ddot{x}=X_{0}(x, t)=\Xi(x)+T(t)
$$


and

$$
\left\{\begin{array}{l}
\Xi(-x)=-\Xi(x) ; \\
T(-t)=T(t), \quad T(t+\pi)=-T(t) .
\end{array}\right.
$$

Corresponding to (1.9), we consider a trigonometric polynomial of the form

$$
x_{m^{\prime}}(t)=\sum_{n=1}^{m^{\prime}} a_{n} \cos (2 n-1) t .
$$

Equality (2.11) implies that

$$
\left\{\begin{array}{l}
x_{m^{\prime}}(-t)=x_{m^{\prime}}(t), \\
x_{m^{\prime}}(t+\pi)=-x_{m^{\prime}}(t) .
\end{array}\right.
$$

Then from (2.10) readily follows that

$$
\left\{\begin{array}{l}
\Xi\left[x_{m^{\prime}}(-t)\right]=\Xi\left[x_{m^{\prime}}(t)\right], \\
\Xi\left[x_{m^{\prime}}(t+\pi)\right]=-\Xi\left[x_{m^{\prime}}(t)\right] .
\end{array}\right.
$$

Now comparing (2.11) with (2.7) we see that

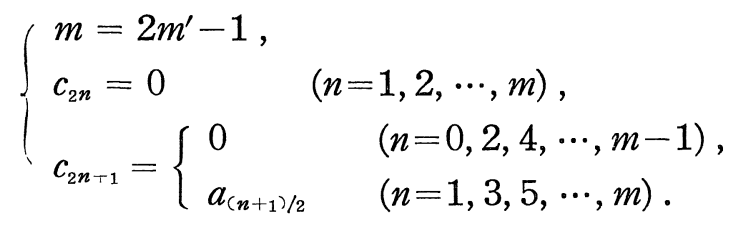

Then by (2.10) and (2.13), we readily see that the determining equation (2.8) can be reduced to the equation

(2.15) $\quad F_{n}(\boldsymbol{a}) \triangleq \frac{1}{\pi} \int_{0}^{2 \pi} X_{0}\left[x_{m^{\prime}}(t), t\right] \cos (2 n-1) t \cdot d t+(2 n-1)^{2} a_{n}=0$

$$
\left(n=1,2, \cdots, m^{\prime}\right),
$$

where $\boldsymbol{a}=\operatorname{col}\left(a_{1}, a_{2}, \cdots, a_{m^{\prime}}\right)$. Equation (2.15) will be called a $d e$ termining equation for Galerkin approximations of the form (2.11) for the second order equation (2.9).

\section{4 Numerical solution of determining equations by Newton's} method. In order to get Galerkin approximations to $2 \pi$-periodic solutions to equation (1.7), it is necessary to solve numerically determining equations of the form (2.8) or (2.15). In the present 
paper, we have solved determining equations (2.8) and (2.15) numerically by Newton's method.

In order to practise Newton's iterative process on a computer, as is seen from (2.8) and (2.15), it is necessary to evaluate Fourier coefficients of known functions on a computer. For this purpose, we have used the following formula [16]:

where

$$
\begin{array}{r}
\frac{1}{\pi} \int_{0}^{2 \pi} f(t)\left\{\begin{array}{c}
\sin p t \\
\cos p t
\end{array}\right\} d t \fallingdotseq \frac{1}{N} \sum_{i=1}^{2 N} f\left(t_{i}\right)\left\{\begin{array}{c}
\sin p t_{i} \\
\cos p t_{i}
\end{array}\right\} \\
(p=0,1,2, \cdots, \nu),
\end{array}
$$

and

$$
t_{i}=\frac{2 i-1}{2 N} \pi \quad(i=1,2, \cdots, 2 N)
$$

In the present paper, for (2.7) and (2.8), we have chosen $m$ and $N$ so that

$$
m=15, \quad N=2^{5}=32,
$$

and, for (2.11) and (2.15), we have chosen $m^{\prime}$ and $N$ so that

$$
m^{\prime}=15, \quad N=2^{6}=64 \text {. }
$$

When we use the formula (2.16) for evaluation of Fourier coefficients appearing in Newton's iterative process, it is necessary to evaluate trigonometric polynomials of the form (2.7) or (2.11) for $t=t_{i}(i=1,2, \cdots, 2 N)$. We have evaluated these trigonometric polynomials by the use of following recurrence formulas.

Recurrence formula 1 [16]. Let

$$
\phi(t)=c_{1}+\sum_{n=1}^{m}\left(c_{2 n} \sin n t+c_{2 n+1} \cos n t\right)
$$

and

$$
\begin{aligned}
\bar{c}_{\mu}=c_{\mu}+2 \bar{c}_{\mu+2} \cos t-\bar{c}_{\mu+4} \\
\left(\begin{array}{l}
\mu=2 m+1,2 m, 2 m-1, \cdots, 3,2,1 ; \\
\bar{c}_{2 m \mid 5}=\bar{c}_{2 m ! 4}=\bar{c}_{2 m+3}=\bar{c}_{2 m \mid 2}=0
\end{array}\right),
\end{aligned}
$$

then

$$
\phi(t)=\bar{c}_{1}+\bar{c}_{2} \sin t-\bar{c}_{3} \cos t .
$$


Recurrence formula 2. Let

$$
\phi(t)=\sum_{n=1}^{m^{\prime}} a_{n} \cos (2 n-1) t
$$

and

$$
\begin{aligned}
\bar{a}_{n}=a_{n}+2 \bar{a}_{n+1} \cos 2 t-\bar{a}_{n+2} \\
\left(\begin{array}{l}
n=m^{\prime}, m^{\prime}-1, \cdots, 2,1 ; \\
\bar{a}_{m^{\prime}+2}=\bar{a}_{m^{\prime}+1}=0
\end{array}\right),
\end{aligned}
$$

then

$$
\phi(t)=\left(\bar{a}_{1}-\bar{a}_{2}\right) \cos t
$$

Recurrence formula 2 can be proved analogously to formula 1 .

2.5 Starting approximations for Newton's iterative process. In order to solve determining equations by Newton's iterative process, it is necessary to find the starting approximate solutions to determining equations.

$1^{\circ}$ Duffing's equation with damping absent. We consider equation (1.7) with $\sigma=0$. In this case, Galerkin approximations under question is of the form (2.11) and the determining equation is of the form (2.15). To find a starting approximate solution for Newton's iterative process, corresponding to (2.11), we consider a Galerkin approximation of the form

$$
\bar{x}(t)=a_{1} \cos t+a_{2} \cos 3 t .
$$

By (2.15), we then have the determining equation as follows.

$$
\left\{\begin{array}{l}
a_{1}\left[(9-\Omega)+\frac{27}{4} \varepsilon\left(a_{1}^{2}+a_{1} a_{2}+2 a_{2}^{2}\right)\right]=0, \\
(1-\Omega) a_{2}+\frac{1}{4} \varepsilon\left(a_{1}^{3}+6 a_{1}^{2} a_{2}+3 a_{2}^{3}\right)-1=0 .
\end{array}\right.
$$

From the first equation of (2.22), we have

$$
a_{1}=0 \quad \text { or }(9-\Omega)+\frac{27}{4} \varepsilon\left(a_{1}^{2}+a_{1} a_{2}+2 a_{2}^{2}\right)=0 \text {. }
$$

Combining these equations with the second equation of (2.22), we have the following two cases. 


\section{Case I.}

$$
\left\{\begin{array}{l}
a_{1}=0 \\
\Omega=1+\frac{3}{4} \varepsilon a_{2}^{2}-\frac{1}{a_{2}}
\end{array}\right.
$$

Case II.

$$
\left\{\begin{array}{l}
\Omega=9+\frac{27}{4} \varepsilon\left(a_{1}^{2}+a_{1} a_{2}+2 a_{2}^{2}\right) \\
51 a_{2}^{3}+27 a_{1} a_{2}^{2}+21 a_{1}^{2} a_{2}-a_{1}^{3}+\frac{4}{\varepsilon}\left(8 a_{2}+1\right)=0 .
\end{array}\right.
$$

In Case II, from the first equation of (2.24), we readily see that real solutions of (2.22) can exist only for

$$
\Omega>9 \text {. }
$$

Now the derivative of the left member of the second equation of (2.24) with respect to $a_{2}$ is always positive, therefore the second equation of (2.24) can have only one real solution $a_{2}$ for any given value of $a_{1}$. Such being the case, for $\varepsilon=1 / 8,1 / 2,1$ and $a_{1}=-5(1) 5$, we have computed $a_{2}$ satisfying the second equation of (2.24) by Newton's method and then we have computed the corresponding values of $\Omega$ using the first equation of (2.24). Making use of the results obtained, we have drawn the graphs of $\left(\Omega, a_{1}\right)$ and $\left(\Omega, a_{2}\right)$ and, from these graphs, we have found the approximate solutions of (2.22) for

$$
\Omega=3.05^{2}, 3.1^{2}, 3.2^{2}, 4^{2} .
$$

Finally, starting from these approximate solutions, we have computed the solutions of $(2.22)$ by Newton's method for values of $\Omega$ specified in (2.26).

In Case I, drawing the graph of $\left(\Omega, a_{2}\right)$ by the use of the second equation of (2.23), we have found the approximate values of $a_{2}$ satisfying the second equation of (2.23) for values of $\Omega$ specified in (2.26). Next, starting from these approximate values of $a_{2}$, by Newton's method, we have computed the values of $a_{2}$ satisfying the second equation of (2.23) for values of $\Omega$ specified in (2.26). 
However, in Case I, we have computed only the values of $a_{2}$ lying near those in Case II.

Figures $1.1,1.2$ and 1.3 show the graphs of $\left(\Omega, a_{1}\right)$ and $\left(\Omega, a_{2}\right)$ in Case II by solid lines and the graphs of $\left(\Omega, a_{2}\right)$ in Case I lying near those in Case II by broken lines for $\varepsilon=1 / 8,1 / 2$ and 1 respectively. Tables $1.1,1.2$ and 1.3 show the solutions of (2.22) obtained in the above way for $\varepsilon=1 / 8,1 / 2$ and 1 respectively.

Let $\left(\bar{a}_{1}, \bar{a}_{2}\right)$ be any one of the values listed in Tables $1.1,1.2$ and 1.3 such that $\bar{a}_{1} \neq 0$. Then, for the determining equation (2.15), we can take

$$
a_{1}=\bar{a}_{1}, \quad a_{2}=\bar{a}_{2}, \quad a_{3}=a_{4}=\cdots=a_{15}=0
$$

for the starting approximate solutions from which Newton's iterative process should be started. Practically, starting from these values, by Newton's iterative process, we have got the solutions to (2.15) shown in the first two columns of Tables 2.1.1, 2.1.2, $\cdots, 2.3 .4$. Clearly these give Fourier coefficients of Galerkin approximations of the form (2.11) to subharmonic solutions of order one-third.

From the values $\left(\bar{a}_{1}, \bar{a}_{2}\right)$ listed in Tables $1.1,1.2$ and $1.3 \mathrm{such}$ that $\bar{a}_{1}=0$, we get in the same way solutions of (2.15) which however slightly differ in the last digits from the solutions shown in the last columns of Tables 2.1.1, 2.1.2, $\cdots, 2.3 .4$. These solutions to (2.15) clearly give Galerkin approximations to $2 \pi$-periodic solution to equation (1.3). However, as is seen from Tables 2.1.1, 2.1.2, .., 2.3.4, these $2 \pi$-periodic solutions to (1.3) are supposed to be again of the form (1.9). Hence Galerkin approximations to these solutions can be computed in the same way as Galerkin approximations to subharmonic solutions (that is, $2 \pi$-periodic solutions to (1.7)) replacing $9 / \Omega$ and $\cos 3 t$ in (1.7) by $1 / \Omega$ and $\cos t$ respectively. The values obtained in this way are shown in the last columns of Tables 2.1.1, 2.1.2, $\cdots, 2.3 .4$. Clearly these give Fourier coefficients of Galerkin approximations of the form $(2.11)$ to $2 \pi / 3$-periodic solutions to (1.7), that is, $2 \pi$-periodic solutions to (1.3) which are nothing else harmonic solutions to the given Duffing's equation. 
$2^{\circ}$ Duffing's equation with damping present. For equation (1.7) with small $|\sigma|>0$, by 2.2, Galerkin approximations are of the form (2.7) and the determining equation is of the form (2.8).

Now Galerkin approximations to $2 \pi$-periodic solutions to equation (1.7) or (1.3) with small $|\sigma|>0$ may be supposed to be close to those to (1.7) or (1.3) with $\sigma=0$, that is, the Galerkin approximations obtained in $1^{\circ}$. Hence, for equation (1.7), one can start Newton's iterative process for the determining equation (2.8) from the value

$$
\begin{cases}c_{2 n}=0 & (n=1,2,3, \cdots, m), \\ c_{2 n+1}= \begin{cases}0 & (n=0,2,4, \cdots, m-1), \\ a_{(n+1) / 2} & (n=1,3,5, \cdots, m),\end{cases} \end{cases}
$$

where $m=15$ and $a_{p}(p=1,2, \cdots, 8)$ are the Fourier coefficients of Galerkin approximations listed in the first two columns of Tables 2.1.1, 2.1.2, $\cdots, 2.3 .4$. Galerkin approximations obtained in this way for $\sigma=2^{-10}=0.0009765625$ are shown in Tables 3.1.1, 3.1.2, $\cdots, 3.2 .2$. Clearly these are Galerking approximations to subharmonic solutions to Duffing's equation with small damping present.

For equation (1.3), one can start Newton's iterative process for the determining equation (2.8) analogously using the Fourier coefficients of Galerkin approximations listed in the last columns of Tables 2.1.1, 2.1.2, $\cdots, 2.3 .4$. Galerkin approximations obtained in this way for $\sigma=2^{-4}=0.0625$ are shown in Table 4 . Clearly these are Galerkin approximations to harmonic solutions to Duffing's equation with small damping present.

\section{Error Estimation of Galerkin Approximations and the Stability of Corresponding Periodic Solutions}

3.1 Basic theorem. Let the symbol $\|\cdots\|$ denote the Euclidean norm of vectors or the corresponding norm of matrices. Then the theorem on which our method of error estimation is based reads as follows.

Theorem 2. In differential system (2.1), suppose that $\boldsymbol{X}(\boldsymbol{x}, t)$ is 
continuously differentiable with respect to $x$ in the region $D \times L$, where $D$ is a given region of the $x$-space and $L$ is the real line.

Assume that (2.1) possesses a periodic approximate solution $\boldsymbol{x}=\overline{\boldsymbol{x}}(t)$ lying inside $D$ such that the multipliers of solutions of the linear homogeneous system

$$
\frac{d \boldsymbol{y}}{d t}=\Psi[\overline{\boldsymbol{x}}(t), t] \boldsymbol{y}
$$

are all different from unity, where $\Psi(\boldsymbol{x}, t)$ is the Jacobian matrix of $\boldsymbol{X}(\boldsymbol{x}, t)$ with respect to $\boldsymbol{x}$.

Let $\Phi(t)$ be a fundamental matrix of (3.1) satisfying the initial condition $\Phi(0)=E$ (E the unit matrix) and $H(t, s)=\left(H_{k l}(t, s)\right.$ ) be a piecewise continuous matrix such that

(3. 2) $H(t, s)=\left\{\begin{array}{lll}\Phi(t)[E-\Phi(2 \pi)]^{-1} \Phi^{-1}(s) & \text { for } & 0 \leqq s \leqq t \leqq 2 \pi, \\ \Phi(t)[E-\Phi(2 \pi)]^{-1} \Phi(2 \pi) \Phi^{-1}(s) & \text { for } & 0 \leqq t<s \leqq 2 \pi\end{array}\right.$

Let $M$ be a positive number such that

$$
\left[2 \pi \cdot \max _{0 \leqq t \leqq 2 \pi} \int_{0}^{2 \pi} \sum_{k, l} H_{k l}^{2}(t, s) d s\right]^{1 / 2} \leqq M
$$

and $r$ be a non-negative number such that

$$
\left\|\frac{d \overline{\boldsymbol{x}}(t)}{d t}-\boldsymbol{X}[\overline{\boldsymbol{x}}(t), t]\right\| \leqq r .
$$

If there exist positive constants $\delta$ and $k<1$ such that

$$
\left\{\begin{array}{cc}
\text { (i) } \quad D_{\delta} \Delta\{\boldsymbol{x} \mid\|\boldsymbol{x}-\boldsymbol{x}(t)\| \leqq \delta \text { for some } t\} \subset D \\
\text { (ii) } \quad\|\Psi(\boldsymbol{x}, t)-\Psi[\overline{\boldsymbol{x}}(t), t]\| \leqq k / M \text { for all } \\
(\boldsymbol{x}, t) \text { satisfying }\|\boldsymbol{x}-\boldsymbol{x}(t)\| \leqq \delta \\
\text { (iii) } \quad M r /(1-k) \leqq \delta
\end{array}\right.
$$

then the given differential system (2.1) possesses one and only one periodic solution $\boldsymbol{x}=\hat{\boldsymbol{x}}(t)$ in $D_{\delta}$ and this is an isolated periodic solution. Moreover, for $\boldsymbol{x}=\hat{\boldsymbol{x}}(t)$, it holds that

$$
\|\overline{\boldsymbol{x}}(t)-\hat{\boldsymbol{x}}(t)\| \leqq M r /(1-k) .
$$

For the proof of the theorem, see [13].

When a Galerkin approximation $\overline{\boldsymbol{x}}_{m}(t)$ has been obtained, as will 
be shown later, for $\overline{\boldsymbol{x}}(t)=\overline{\boldsymbol{x}}_{m}(t)$ one can easily find the numbers $M$ and $r$ satisfying (3.3) and (3.4) respectively. Then, as will be illustrated with an example, one can easily check the existence of the constants $\delta$ and $k$ satisfying the condition (3.5). If there exist such constants $\delta$ and $k$, then by the above theorem one can know the existence of an exact periodic solution of (2.1) and, in addition, by (3.6) one can find an error bound to the Galerkin approximation $\overline{\boldsymbol{x}}_{m}(t)$.

As is seen from (3.2) and (3.3), in order to find the number $M$ satisfying (3.3), one has to compute a fundamental matrix $\Phi(t)$ of (3.1) satisfying the initial condition $\Phi(0)=E$. If $\overline{\boldsymbol{x}}(t)$ is close to the exact solution $\hat{\boldsymbol{x}}(t)$, then the eigenvalues of $\Phi(2 \pi)$ is close to the multipliers of solutions of the first variation equation of (2.1) with respect to the exact periodic solution. Hence one may decide the stability of the exact periodic solution by inspecting the absolute values of eigenvalues of the matrix $\Phi(2 \pi)$. In Tables 2.1.1, 2.1.2, $\cdots$, 2.3.4, 3.1.1, $\cdots, 3.2 .2$ and 4 , eigenvalues of $\Phi(2 \pi)$ are shown under the sign $\lambda_{i}(i=1,2)$.

3.2 The number $\boldsymbol{r}$. For equation (1.7) with $\sigma \neq 0$, Galerkin approximations are of the form (2.7). Therefore, as is seen from (2.6), the number $r$ is a number such that

$$
\left|\ddot{x}_{m}(t)-X\left[x_{m}(t), \dot{x}_{m}(t), t\right]\right| \leqq r .
$$

Let $\bar{x}_{m}(t)$ be a Galerkin approximation obtained and let

$$
\begin{aligned}
& \ddot{x}_{m}(t)-X\left[\bar{x}_{m}(t), \dot{\bar{x}}_{m}(t), t\right] \\
& \quad=C_{1}+\sum_{n=1}^{\infty}\left(C_{2 n} \sin n t+C_{2 n+1} \cos n t\right) .
\end{aligned}
$$

Then inequality (3.7) is valid if

$$
\left|C_{1}\right|+\sum_{n=1}^{m_{1}}\left[C_{2 n}^{2}+C_{2 n+1}^{2}\right]^{1 / 2}<r
$$

with large $m_{1}$. In our computations, we have chosen $m_{1}$ so that

$$
m_{1}=25
$$

and, for computation of $C_{1}, C_{2}, \cdots, C_{2 m_{1}+1}$, we have used the formula 
(2.16) with $N=32$. By inequality (3.9), for $r$, we have taken a a number slightly greater than the quantity

$$
\left|C_{1}\right|+\sum_{n=1}^{25}\left[C_{2 n}^{2}+C_{2 n+1}^{2}\right]^{1 / 2}
$$

For equation (1.7) with $\sigma=0$, Galerkin approximations are of the form (2.11). Hence corresponding to a Galerkin approximation $\bar{x}_{m}(t)$ obtained, we have the following expansion instead of (3.8):

$$
\ddot{\bar{x}}_{m}(t)-X_{0}\left[\bar{x}_{m}(t), t\right]=\sum_{n=1}^{\infty} A_{n} \cos (2 n-1) t .
$$

Then inequality (3.7) is valid if

$$
\sum_{n=1}^{m_{1}}\left|A_{n}\right|<r
$$

with large $m_{1}$. In our computations, we have chosen $m_{1}$ so that

$$
m_{1}=25 \text {, }
$$

and, for computation of $A_{1}, A_{2}, \cdots, A_{25}$, we have used the formula (2.16) with $N=64$. By inequality (3.11), for $r$, we have taken a number slightly greater than the quantity

$$
\sum_{n=1}^{25}\left|A_{n}\right|
$$

The above method applies also to equation (1.3) without any change.

In Tables $2.1 .1,2.1 .2, \cdots, 2.3 .4,3.1 .1, \cdots, 3.2 .2$ and 4 , are shown the numbers $r$ found by the above method.

3. 3 The number $M$. To find the number $M$ corresponding to a Galerkin approximation $\bar{x}_{m}(t)$, we first have to compute a fundamental matrix $\Phi(t)$ of (3.1) with $\bar{x}(t)=\bar{x}_{m}(t)$ satisfying the initial condition $\Phi(0)=E$. In the present paper, by the method developed in [14] and [15], we have computed the desired fundamental matrix in the form

$$
\Phi(t) \fallingdotseq \frac{1}{2} B_{0}+\sum_{n=1}^{30} B_{n} T_{n}\left(\frac{t}{\pi}-1\right),
$$

where $T_{n}(t)(n=1,2, \cdots, 30)$ are Chebyshev polynomials such that 


$$
T_{n}(\cos \theta)=\cos n \theta .
$$

By means of (3.2), we then compute

$$
\begin{aligned}
& H(p \pi / 128, q \pi / 128) \\
& \left(\begin{array}{l}
p=0,2,4, \cdots, 256 ; \\
q=0,1,2, \cdots, 256
\end{array}\right) .
\end{aligned}
$$

Making use of $H(p \pi / 128, q \pi / 128)$ obtained, we compute the integrals

$$
\int_{0}^{2 \pi} \sum_{k, l} H_{k l}^{2}(p \pi / 128, s) d s \quad(p=0,2,4, \cdots, 256)
$$

by Simpson's rule with mesh size $\pi / 128$. Then, by (3. 3), a number

$$
\begin{aligned}
{\left[2 \pi \cdot \max _{p} \int_{0}^{2 \pi} \sum_{k, l} H_{k l}^{2}(p \pi / 128, s) d s\right]^{1 / 2} } & (p=0,2,4, \cdots, 256)
\end{aligned}
$$

will give the desired number $M$.

The numbers $M$ calculated in the above way are shown in Tables 2.1.1, 2.1.2, $\cdots, 2.3 .4,3.1 .1, \cdots, 3.2 .2$ and 4 .

3. 4 The numbers $\delta$ and $\boldsymbol{k}$. We shall illustrate with an example how the existence of the numbers $\delta$ and $k$ satisfying the condition (3.5) of Theorem 2 can be checked for Galerkin approximations to periodic solutions to the equation of the form (1.7) or (1.3).

Example. Equation (1.7) with $\sigma=2^{-10}, \varepsilon=1, \omega=3.1$.

By Table 3.2.1, we have two Galerkin approximations, of which the following one will be brought into consideration:

$$
\begin{aligned}
\bar{x}(t)=\bar{x}_{15}(t) & =0.0037484022 \sin t \quad+0.3159324676 \cos t \\
& +0.0000753137 \sin 3 t-0.1174126343 \cos 3 t \\
& -0.0000095962 \sin 5 t-0.0002162865 \cos 5 t \\
& +0.0000006531 \sin 7 t+0.0000637792 \cos 7 t \\
& +0.0000000195 \sin 9 t-0.0000045873 \cos 9 t \\
& -0.0000000014 \sin 11 t-0.0000000458 \cos 11 t \\
& +0.0000000050 \cos 13 t \\
& -0.0000000002 \cos 15 t .
\end{aligned}
$$

Equation (1.7) is evidently equivalent to the first order system 


$$
\left\{\begin{array}{l}
\frac{d x}{d t}=y \\
\frac{d y}{d t}=-\frac{3 \sigma}{\omega} y-\frac{9}{\omega^{2}}\left(x+\varepsilon x^{3}\right)+\frac{9}{\omega^{2}} \cos 3 t
\end{array}\right.
$$

The Jacobian matrix $\Psi(x, y, t)$ of the right member of (3.14) with respect to $x$ and $y$ is

$$
\Psi(x, y, t)=\left[\begin{array}{cc}
0 & 1 \\
-\frac{9}{\omega^{2}}\left(1+3 \varepsilon x^{2}\right) & -\frac{3 \sigma}{\omega}
\end{array}\right],
$$

therefore we have

$$
\|\Psi(x, y, t)-\Psi[\bar{x}(t), \bar{y}(t), t]\|=\frac{27}{\omega^{2}} \varepsilon\left|x^{2}-\bar{x}^{2}(t)\right|,
$$

where $\bar{y}(t)=\dot{\bar{x}}(t)$. From (3.15), for

$$
\left[|x-\bar{x}(t)|^{2}+|y-\bar{y}(t)|^{2}\right]^{1 / 2} \leqq \delta,
$$

we then have

$$
\|\Psi(x, y, t)-\Psi[\bar{x}(t), \bar{y}(t), t]\| \leqq \frac{27}{\omega^{2}} \varepsilon \delta[\delta+2|\bar{x}(t)|],
$$

therefore we see that for system (3.14), the condition (3.5) is satisfied by the numbers $\delta$ and $k$. They satisfy

$$
\left\{\begin{array}{l}
\frac{27}{\omega^{2}} \varepsilon \delta[\delta+2 \cdot \max |\bar{x}(t)|] \leqq \frac{k}{M}, \\
\frac{M r}{1-k} \leqq \delta
\end{array}\right.
$$

where $r$ and $M$ are numbers specified in Theorem 2 for Galerkin approximation (3.13). By Table 3.2.1, we see that

$$
r=2.9 \times 10^{-9}, \quad M=32.1 \text {. }
$$

From (3.13), we readily see that

$$
\begin{aligned}
& |\bar{x}(t)| \leqq\left[0.0037484022^{2}+0.3159324676^{2}\right]^{1 / 2} \\
& +\left[0.00007 \cdot 53137^{2}+0.1174126343^{2}\right]^{1 / 2}
\end{aligned}
$$

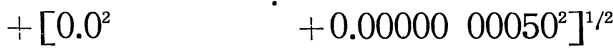

$$
\begin{aligned}
& +\left[0.0^{2}+0.0000000002^{2}\right]^{1 / 2} \\
& =0.4336522820 \text {. }
\end{aligned}
$$


Hence substituting $\varepsilon=1, \omega=3.1$ and (3.19) into (3.18), we see that inequality (3.18) is valid if

$$
\left\{\begin{array}{l}
\frac{27}{9.61} \times \delta \times(\delta+0.8673045640) \leqq \frac{k}{32.1}, \\
\frac{32.1 \times 2.9 \times 10^{-9}}{1-k} \leqq \delta
\end{array}\right.
$$

The second inequality of (3.20) means

$$
\frac{9.309 \times 10^{-8}}{1-k} \leqq \delta .
$$

Now we expect $k \ll 1$. Therefore taking into account inequality (3.21), we assume that

$$
\delta \leqq 1 \times 10^{-7} .
$$

Then the first inequality of (3.20) is valid if

$$
\frac{27}{9.61} \times 0.8673046640 \times \delta \leqq \frac{k}{32.1},
$$

that is,

$$
\begin{aligned}
\delta & \leqq \\
& =0.01278 \quad 44 \cdots \times k .
\end{aligned}
$$

Hence we suppose that

$$
\delta \leqq 0.01278 k \text {. }
$$

Then combining (3.23) with (3.21), we have

$$
\frac{9.309 \times 10^{-8}}{1-k} \leqq \delta \leqq 0.01278 k \text {. }
$$

Now from

$$
\frac{9.309 \times 10^{-8}}{1-k} \leqq 0.01278 k,
$$

we have

$$
9.309 \times 10^{-8} \leqq 0.01278 k(1-k),
$$

that is,

$$
\frac{9.309 \times 10^{-8}}{0.01278}=7.284037 \cdots \times 10^{-6} \leqq k(1-k) .
$$


Since we expect small $k$, we suppose that

$$
k=8 \times 10^{-6} \text {. }
$$

For this value of $k$,

$$
\left\{\begin{array}{l}
\frac{9.309 \times 10^{-8}}{1-k}=9.3090744 \cdots \times 10^{-8} \\
0.01278 k=10.224 \cdots \times 10^{-8}
\end{array}\right.
$$

Hence taking into account the assumption (3.22), from (3.24) and (3.25), we see that inequality (3.20) is valid for

$$
k=8 \times 10^{-6} \text { and } 9.30908 \times 10^{-8} \leqq \delta \leqq 1 \times 10^{-7},
$$

in other words, the condition (3.5) of Theorem 2 is satisfied by the numbers $\delta$ and $k$ specified in (3.26).

By Theorem 2, we thus see that equation (1.7) with $\sigma=2^{-10}$, $\varepsilon=1, \omega=3.1$ possesses a unique periodic solution $\hat{x}(t)$ in the region

$$
\left[x-\bar{x}|(t)|^{2}+|\dot{x}-\dot{\bar{x}}(t)|^{2}\right]^{1 / 2} \leqq \delta_{0}=1 \times 10^{-7}
$$

and moreover

$$
\left[|\bar{x}(t)-\hat{x}(t)|^{2}+|\dot{\bar{x}}(t)-\dot{\hat{x}}(t)|^{2}\right]^{1 / 2} \leqq E=9.31 \times 10^{-8} .
$$

Remark. All Galerkin approximations listed in Tables 2.1.1, $2.1 .2, \cdots, 2.3 .4,3.1 .1, \cdots, 3.2 .2$ and 4 satisfy the condition of Theorem 2. Therefore corresponding to each Galerkin approximation listed in these tables, an exact periodic solution exists. The error bounds $E$ to Galerkin approximations obtained by the application of Theorem 2 are shown in Tables 2.1.1, 2.1.2, $\cdots, 2.3 .4,3.1 .1, \cdots$, 3.2.2 and 4. In Tables 3.1.1, $\cdots, 3.2 .2$ and 4 , the numbers $\delta_{0}$ which, as in (3.27), fix the region of the existence of exact periodic solutions, are also shown.

\section{Conclusions}

By Tables 2.1.1, 2.1.2, $\cdots, 2.3 .4$, we see that for $\varepsilon=1 / 8,1 / 2$, 1 and $\omega=3.05,3.1,3.2,4$, Duffing's equation with damping absent possesses a harmonic solution with the neutral stability and two kinds 
of subharmonic solutions, of which one has the neutral stability and the other is unstable. For Duffing's equation with damping absent, subharmonic solutions with the neutral stability will be called subharmonic solutions of the first kind and unstable subharmonic solutions will be called subharmonic solutions of the second kind. By Tables 3.1.1, 3.1.2, $\cdots, 3.2 .2$ and 4 , we further see that for $\varepsilon=1 / 8,1$ and $\omega=3.1,4$, Duffing's equation with small positive damping present possesses a stable harmonic solution and two kinds of subharmonic solutions, of which one close to subharmonic solutions of the first kind of the equation with damping absent is stable and the other close to subharmonic solutions of the second kind is unstable.

From Tables 2.1.1, 2.1.2, $\cdots, 2.3 .4,3.1 .1, \cdots, 3.2 .2$ and 4 , we further observe some properties of periodic solutions to Duffing's equation. They will be stated in the following sections.

4. 1 Symmetricity of exact periodic solutions.

$1^{\circ}$ Periodic solutions to Duffing's equation with damping absent. In Tables 2.1.1, 2.1.2, $\cdots, 2.3 .4$, every Galerkin approximation to a subharmonic solution, that is, a $2 \pi$-periodic solution to (1.7) with $\sigma=0$ and one to a harmonic solution, that is, a $2 \pi$-periodic solution to (1.3) with $\sigma=0$ are both of the form

$$
\bar{x}(t)=\sum_{n=1}^{m} a_{n} \cos (2 n-1) t .
$$

Let $\hat{x}(t)$ be a corresponding exact periodic solution, then by Theorem 2 we have

$$
\left[|\hat{x}(t)-\bar{x}(t)|^{2}+\left|\hat{x}^{\prime}(t)-\bar{x}^{\prime}(t)\right|^{2}\right]^{1 / 2} \leqq E \leqq \delta_{0},
$$

where ' denotes the differentiation with respect to the argument. Now from (4.1) we have

$$
\bar{x}(-t)=-\bar{x}(t+\pi)=\bar{x}(t),
$$

therefore from (4.2) we have

$$
\begin{aligned}
& {\left[|\hat{x}(-t)-\bar{x}(t)|^{2}+\left|\frac{d}{d t} \hat{x}(-t)-\bar{x}^{\prime}(t)\right|^{2}\right]^{1 / 2} } \\
= & {\left[|\hat{x}(-t)-\bar{x}(-t)|^{2}+\left|-\hat{x}^{\prime}(-t)+\bar{x}^{\prime}(-t)\right|^{2}\right]^{1 / 2} } \\
= & {\left[|\hat{x}(-t)-\bar{x}(-t)|^{2}+\left|\hat{x}^{\prime}(-t)-\bar{x}^{\prime}(-t)\right|^{2}\right]^{1 / 2} } \\
\leqq & E \leqq \delta_{0}
\end{aligned}
$$


and

$$
\begin{aligned}
& {\left[|-\hat{x}(t+\pi)-\bar{x}(t)|^{2}+\left|-\frac{d}{d t} \hat{x}(t+\pi)-\bar{x}^{\prime}(t)\right|^{2}\right]^{1 / 2} } \\
= & {\left[|-\hat{x}(t+\pi)+\bar{x}(t+\pi)|^{2}+\left|-\hat{x}^{\prime}(t+\pi)+\bar{x}^{\prime}(t+\pi)\right|^{2}\right]^{1 / 2} } \\
\leqq & E \leqq \delta_{0} .
\end{aligned}
$$

However from the symmetricity of equations (1.7) and (1.3), $\hat{x}(-t)$ and $-\hat{x}(t+\pi)$ are also periodic solutions to (1.7) or (1.3) correspondingly. Then, since a periodic solution to (1.7) or (1.3) satisfying inequality (4.2) is unique by Theorem 2 , we see that

$$
\hat{x}(-t)=-\hat{x}(t+\pi)=\hat{x}(t),
$$

which means that the Fourier series of $\hat{x}(t)$ is of the form

$$
\hat{x}(t)=\sum_{n=1}^{\infty} \hat{a}_{n} \cos (2 n-1) t .
$$

$2^{\circ}$ Periodic solutions to Duffing's equation with damping present. In Tables 3.1.1, 3.1.2, 3.2.1 and 3.2.2, every Galerkin approximation $\bar{x}(t)$ to a subharmonic solution, that is, a $2 \pi$-periodic solution to (1.7) with $\sigma \neq 0$ satisfies the inequality

$$
\left[|\bar{x}(t+\pi)+\bar{x}(t)|^{2}+\left|\bar{x}^{\prime}(t+\pi)+\bar{x}^{\prime}(t)\right|^{2}\right]^{1 / 2} \leqq \sqrt{52} \times 10^{-10} .
$$

Let $\hat{x}(t)$ be a corresponding exact periodic solution, then by Theorem 2 we have

$$
\left[|\hat{x}(t)-\bar{x}(t)|^{2}+\left|\hat{x}^{\prime}(t)-\bar{x}^{\prime}(t)\right|^{2}\right]^{1 / 2} \leqq E .
$$

Then from (4.6) and (4.5) we readily get

$$
\begin{aligned}
& {\left[|\hat{x}(t+\pi)+\bar{x}(t)|^{2}+\left|\hat{x}^{\prime}(t+\pi)+\bar{x}^{\prime}(t)\right|^{2}\right]^{1 / 2} } \\
\leqq & {\left[|\hat{x}(t+\pi)-\bar{x}(t+\pi)|^{2}+\left|\hat{x}^{\prime}(t+\pi)-\bar{x}^{\prime}(t+\pi)\right|^{2}\right]^{1 / 2} } \\
+ & {\left[|\bar{x}(t+\pi)+\bar{x}(t)|^{2}+\left|\bar{x}^{\prime}(t+\pi)+\bar{x}^{\prime}(t)\right|^{2}\right]^{1 / 2} } \\
\leqq & E+\sqrt{52} \times 10^{-10} .
\end{aligned}
$$

However, as is seen from Tables $3.1 .1,3.1 .2,3.2 .1$, and 3.2.2,

$$
E+\sqrt{52} \times 10^{-10}<E+8 \times 10^{-10}<\delta_{0},
$$

which by (4.7) implies

$$
\left[|\hat{x}(t+\pi)+\bar{x}(t)|^{2}+\left|\hat{x}^{\prime}(t+\pi)+\bar{x}^{\prime}(t)\right|^{2}\right]^{1 / 2}<\delta_{0} .
$$


Now from the symmetricity of equation (1.7), $-\hat{x}(t+\pi)$ is also a periodic solution of (1.7). Then, since a periodic solution of (1.7) satisfying the inequality

$$
\left[|\hat{x}(t)-\bar{x}(t)|^{2}+\left|\hat{x}^{\prime}(t)-\bar{x}^{\prime}(t)\right|^{2}\right]^{1 / 2} \leqq \delta_{0}
$$

is unique by Theorem 2 , we see that

$$
\hat{x}(t+\pi)=-\hat{x}(t),
$$

which means that the Fourier series of $\hat{x}(t)$ is of the form

$$
\hat{x}(t)=\sum_{n=1}^{\infty}\left[\hat{c}_{2 n} \sin (2 n-1) t+\hat{c}_{2 n+1} \cos (2 n-1) t\right] .
$$

For all harmonic solutions $\hat{x}(t)$ corresponding to Galerkin approximations listed in Table 4, it can be proved in the way similar to $1^{\circ}$ that $\hat{x}(t)$ can be expanded in Fourier serics of the form (4.9).

4. 2 Remarkable character of periodic solutions to Duffing's equation with damping absent. Tables $2.1 .1,21.2, \cdots, 2.3 .4$ show that in the Fourier series of the subharmonic solutions, the first two coefficients $a_{1}$ and $a_{2}$ dominate remaining ones strongly and, in the Fourier series of the harmonic solutions, the first coefficient $a_{2}$ dominates remaining ones strongly. Comparing Tables 2.1.1, 2.1.2, $\cdots, 2.3 .4$ with Tables $1.1,1.2,1.3$, we further see that the above dominant Fourier coefficients of the subharmonic solutions and the harmonic solutions are all very close to the values of $a_{1}$ and $a_{2}$ listed in Tables 1.1, 1.2, 1.3, that is, the Fourier coefficients of Galerkin approximations of the form

$$
\bar{x}(t)=a_{1} \cos t+a_{2} \cos 3 t .
$$

This implies that even for non-small $\varepsilon>0$, one can know the qualitative character of periodic solutions to Duffing's equation with damping absent by investigating the character of the Galerkin approximations of the form (4.10). Then we may suppose that Figures 1.1, 1.2 and 1.3 are valid also for periodic solutions to Duffing's equation with damping absent. 
4. 3 A remark to periodic solutions to Duffing's equation with damping present. In 4.1, we have observed that periodic solutions to Duffing's equation with small damping present are of the form

$$
x(t)=\sum_{n=1}^{\infty} b_{n} \sin (2 n-1) t+\sum_{n=1}^{\infty} c_{n} \cos (2 n-1) t .
$$

Comparing Tables 3.1.1, 3.1.2, 3.2.1, 3.2.2 and 4 with Tables 2.1.2, $2.1 .4,2.3 .2,2.3 .4$, we observe that

$$
\sum_{n=1}^{\infty} c_{n} \cos (2 n-1) t \fallingdotseq \sum_{n=1}^{\infty} a_{n} \cos (2 n-1) t,
$$

where $\sum_{n=1}^{\infty} a_{n} \cos (2 n-1) t$ is a corresponding periodic solution to Duffing's equation with damping absent. In Tables 3.1.1 and 3.1.2, we further observe that for $\varepsilon=1 / 8$,

$$
\sum_{n=1}^{\infty} b_{n} \sin (2 n-1) t
$$

is not a small quantity of the order $\sigma=2^{-10}=0.0009765625$. 
Fig. $1.1(\varepsilon=1 / 8)$.

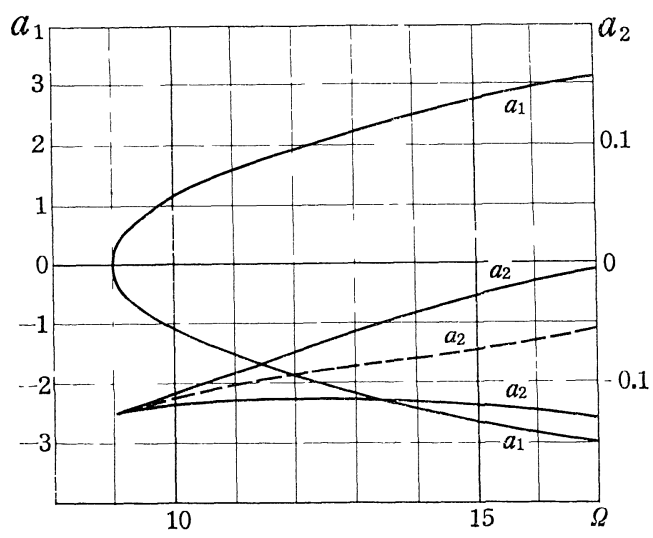

Fig. $1.2(\varepsilon=1 / 2)$.

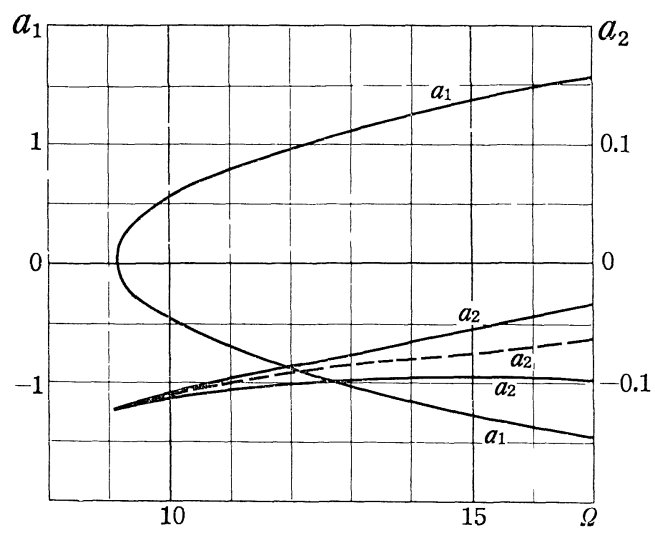

Fig. $1.3(\varepsilon=1)$

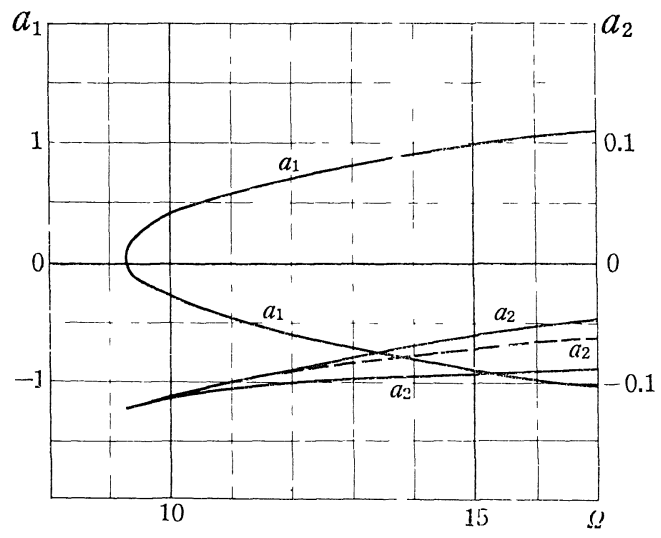


Table $1.1(\varepsilon=1 / 8)$

\begin{tabular}{|c|c|c|c|}
\hline$\omega$ & $\Omega$ & $a_{1}$ & $a_{2}$ \\
\hline 3.05 & 9.3025 & $\begin{array}{l}0.637418236 \\
0.0 \\
--0.515852082\end{array}$ & $\begin{array}{l}-0.120597225 \\
-0.120465389 \\
-0.121714132\end{array}$ \\
\hline 3.1 & 9.61 & $\begin{array}{ll} & 0.894201639 \\
0.0 & \\
-0.77575 & 5977\end{array}$ & $\begin{array}{l}-0.115578278 \\
-0.116161085 \\
-0.119422055\end{array}$ \\
\hline 3.2 & 10.24 & $\begin{array}{l}1.256759007 \\
0.0 \\
-1.144263122\end{array}$ & 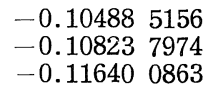 \\
\hline 4.0 & 16.0 & $\begin{array}{l}2.889389313 \\
0.0 \\
-2.812819167\end{array}$ & $\begin{array}{ll}-0.01832 & 4240 \\
-0.06666 & 8519 \\
-0.12545 & 0320\end{array}$ \\
\hline
\end{tabular}

Table $1.2(\varepsilon=1 / 2)$.

\begin{tabular}{|c|c|c|c|}
\hline$\omega$ & $\Omega$ & $a_{1}$ & $a_{2}$ \\
\hline 3.05 & 9.3025 & $\begin{array}{l}0.313451389 \\
0.0 \\
-0.192446982\end{array}$ & $\begin{array}{r}-0.121137420 \\
-0.120524726 \\
-0.121037994\end{array}$ \\
\hline 3.1 & 9.61 & $\begin{array}{ll} & 0.454461607 \\
& 0.0 \\
- & 0.336510725\end{array}$ & 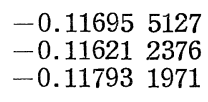 \\
\hline 3.2 & 10.24 & $\begin{array}{ll} & 0.643119357 \\
& 0.0 \\
- & 0.531009109\end{array}$ & $\begin{array}{l}-0.108314549 \\
-0.108276627 \\
-0.11289 \\
2860\end{array}$ \\
\hline 4.0 & 16.0 & $\begin{array}{l}1.461659437 \\
0.0 \\
-1.385154020\end{array}$ & 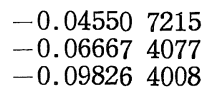 \\
\hline
\end{tabular}

Table $1.3(\varepsilon=1)$

\begin{tabular}{|c|c|c|c|}
\hline$\omega$ & $\Omega$ & $a_{1}$ & $a_{2}$ \\
\hline 3.05 & 9.3025 & $\begin{array}{l}0.198795839 \\
0.0 \\
-0.078537428\end{array}$ & $\begin{array}{ll}-0.12123 & 5672 \\
-0.12060 & 4116 \\
-0.12075 & 3860\end{array}$ \\
\hline 3.1 & 9.61 & $\begin{array}{l}0.316088307 \\
0.0 \\
-0.198794931\end{array}$ & $\begin{array}{ll}-0.11741 & 1722 \\
-0.11628 & 0975 \\
-0.11732 & 0538\end{array}$ \\
\hline 3.2 & 10.24 & $\begin{array}{l}0.458135606 \\
0.0 \\
-0.346538367\end{array}$ & 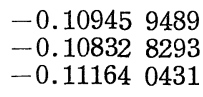 \\
\hline 4.0 & 16.0 & $\begin{array}{l}1.042682507 \\
0.0 \\
-0.966263409\end{array}$ & $\begin{array}{ll}-0.05360 & 9552 \\
-0.06668 & 1491 \\
-0.09015 & 7062\end{array}$ \\
\hline
\end{tabular}


Table 2.1.1

Periodic solutions to (1.7) with $\sigma=0 \quad(\varepsilon=1 / 8, \omega=3.05)$

\begin{tabular}{|c|c|c|c|}
\hline \multirow[b]{2}{*}{$n$} & \multicolumn{2}{|c|}{ Subharmonic solutions } & \multirow{2}{*}{$\begin{array}{c}\text { Harmonic solution } \\
a_{n}\end{array}$} \\
\hline & $a_{n}$ & $a_{n}$ & \\
\hline $\begin{array}{c}1 \\
2 \\
3 \\
4 \\
5 \\
6 \\
7 \\
8 \\
9 \\
\vdots \\
\vdots \\
15\end{array}$ & $\begin{array}{rr}0.63738 & 94700 \\
-0.12059 & 77217 \\
-0.00015 & 03858 \\
0.00001 & 74607 \\
-0.00000 & 06320 \\
-0.00000 & 00038 \\
0.00000 & 00002 \\
0.0 & \\
\vdots \\
\vdots \\
\vdots \\
0.0\end{array}$ & $\begin{array}{cc}-0.51581 & 55306 \\
-0.12171 & 46120 \\
-0.00015 & 14040 \\
-0.00001 & 45577 \\
-0.00000 & 07096 \\
-0.00000 & 00033 \\
-0.00000 & 00002 \\
0.0 & \\
\vdots & \\
\vdots & \\
\vdots & \\
0.0 & \end{array}$ & $\begin{array}{ll}0.0 & \\
-0.12046 & 53891 \\
0.0 & \\
0.0 & \\
-0.00000 & 06604 \\
0.0 & \\
\vdots \\
\vdots \\
\vdots \\
\vdots \\
0.0\end{array}$ \\
\hline$r$ & $8.63 \times 10^{-9}$ & $1.31 \times 10^{-8}$ & $5 \times 10^{-10}$ \\
\hline$M$ & 101.5 & 123.7 & 9.9 \\
\hline$E$ & $8.760 \times 10^{-7}$ & $1.6207 \times 10^{-6}$ & $5.0 \times 10^{-9}$ \\
\hline$\lambda_{i}$ & $\begin{array}{l}0.9932102880 \\
\quad \pm 0.1163328121 i\end{array}$ & $\begin{array}{ll}1.09964 & 4524, \\
0.90938 & 47852 \\
\end{array}$ & $\begin{array}{l}-0.4724471969 \\
\pm 0.8813589769 i\end{array}$ \\
\hline$\left|\lambda_{i}\right|$ & 0.9999999996 & & 1.000000000 \\
\hline Stability & neutral & unstable & neutral \\
\hline
\end{tabular}

Table 2.1.2

Periodic solutions to (1.7) with $\sigma=0(\varepsilon=1 / 8, \omega=3.1)$

\begin{tabular}{|c|c|c|c|}
\hline & Subharmoni & solutions & Harmonic solution \\
\hline$n$ & $a_{n}$ & $a_{n}$ & $a_{n}$ \\
\hline $\begin{array}{c}1 \\
2 \\
3 \\
4 \\
5 \\
6 \\
7 \\
8 \\
9 \\
\vdots \\
\vdots \\
15\end{array}$ & $\begin{array}{rr}0.89416 & 37367 \\
-0.11558 & 04311 \\
-0.00029 & 52928 \\
0.00002 & 15640 \\
-0.00000 & 04842 \\
-0.00000 & 00063 \\
0.00000 & 00002 \\
0.0 & \\
\vdots & \\
\vdots & \\
\vdots & \\
0.0 & \end{array}$ & $\begin{array}{cc}-0.77570 & 72093 \\
-0.11942 & 42151 \\
-0.00030 & 39846 \\
-0.00002 & 06942 \\
-0.00000 & 07033 \\
-0.00000 & 00064 \\
-0.00000 & 00002 \\
0.0 & \\
\vdots & \\
\vdots & \\
\vdots & \\
0.0 & \end{array}$ & $\begin{array}{ll}0.0 & \\
-0.11616 & 10854 \\
0.0 & \\
0.0 & \\
-0.00000 & 05730 \\
0.0 & \\
\vdots \\
\vdots \\
\vdots \\
\vdots \\
0.0\end{array}$ \\
\hline$r$ & $9.6 \times 10^{-9}$ & $1.86 \times 10^{-8}$ & $5 \times 10^{-10}$ \\
\hline$M$ & 76.1 & 86.0 & 10.2 \\
\hline$E$ & $7.306 \times 10^{-7}$ & $1.5998 \times 10^{-6}$ & $5.2 \times 10^{-9}$ \\
\hline$\lambda_{i}$ & $\begin{array}{r}0.9826741279 \\
\quad+0.18534 \quad 17320 i \\
\end{array}$ & $\begin{array}{ll}1.17720 & 8198 \\
0.84946 & 74103\end{array}$ & $\begin{array}{r}-0.4426932662 \\
\pm 0.8966731133 i\end{array}$ \\
\hline$\left|\lambda_{2}\right|$ & 0.9999999997 & & 1.000000000 \\
\hline Stability & neutral & unstable & neutral \\
\hline
\end{tabular}


Table 2.1.3

Periodic solutions to (1.7) with $\sigma=0 \quad(\varepsilon=1 / 8, \omega=3.2)$

\begin{tabular}{|c|c|c|c|}
\hline \multirow[b]{2}{*}{$n$} & \multicolumn{2}{|c|}{ Subharmonic solutions } & \multirow{2}{*}{$\frac{\text { Harmonic solution }}{a_{n}}$} \\
\hline & $a_{n}$ & $a_{n}$ & \\
\hline $\begin{array}{c}1 \\
2 \\
3 \\
4 \\
5 \\
6 \\
7 \\
8 \\
9 \\
\vdots \\
\vdots \\
15\end{array}$ & $\begin{array}{rr}1.25671 & 76442 \\
-0.10489 & 29084 \\
-0.00052 & 42595 \\
0.00002 & 26175 \\
-0.00000 & 02233 \\
-0.00000 & 00080 \\
0.00000 & 00001 \\
0.0 & \\
\vdots & \\
\vdots & \\
\vdots & \\
0.0 & \end{array}$ & $\begin{array}{cc}-1.14419 & 82013 \\
-0.11640 & 92077 \\
-0.00057 & 89532 \\
-0.00002 & 82413 \\
-0.00000 & 07476 \\
-0.00000 & 00117 \\
-0.00000 & 00003 \\
0.0 & \\
\vdots & \\
\vdots & \\
\vdots & \\
0.0 & \end{array}$ & $\begin{array}{ll}0.0 & \\
-0.10823 & 79741 \\
0.0 & \\
0.0 & \\
-0.00000 & 04347 \\
0.0 & \\
\vdots \\
\vdots \\
\vdots \\
\vdots \\
0.0\end{array}$ \\
\hline$r$ & $1.68 \times 10^{-8}$ & $1.71 \times 10^{-8}$ & $2 \times 10^{-10}$ \\
\hline$M$ & 61.8 & 66.2 & 10.8 \\
\hline$E$ & $1.0384 \times 10^{-6}$ & $1.1321 \times 10^{-6}$ & $2.2 \times 10^{-9}$ \\
\hline$\lambda_{i}$ & $\begin{array}{l}0.9602145254 \\
\quad \pm 0.27926 \quad 34323 i\end{array}$ & $\begin{array}{l}1.297452642, \\
0.77074 \quad 10404\end{array}$ & $\begin{array}{l}-0.3846739051 \\
\quad \pm 0.9230525373 i\end{array}$ \\
\hline$\left|\lambda_{i}\right|$ & 0.9999999997 & & 1.000000000 \\
\hline Stability & neutral & unstable & neutral \\
\hline
\end{tabular}

Table 2.1.4

Periodic solutions to (1.7) with $\sigma=0 \quad(\varepsilon=1 / 8, \omega=4)$

\begin{tabular}{|c|c|c|c|}
\hline \multirow[b]{2}{*}{$n$} & \multicolumn{2}{|c|}{ Subharmonic solutions } & \multirow{2}{*}{$\frac{\text { Harmonic solution }}{a_{n}}$} \\
\hline & $a_{n}$ & $a_{n}$ & \\
\hline $\begin{array}{c}1 \\
2 \\
3 \\
4 \\
5 \\
6 \\
7 \\
8 \\
9 \\
\vdots \\
\vdots \\
15\end{array}$ & $\begin{array}{cc}2.88939 & 64903 \\
-0.01834 & 34265 \\
-0.00034 & 06838 \\
-0.00000 & 20357 \\
0.00000 & 00117 \\
0.00000 & 00002 \\
0.0 & \\
\vdots \\
\vdots \\
\vdots \\
\vdots \\
0.0\end{array}$ & $\begin{array}{cc}-2.81264 & 00834 \\
-0.12557 & 78220 \\
-0.00232 & 01890 \\
-0.00007 & 13067 \\
-0.00000 & 19406 \\
-0.00000 & 00528 \\
-0.00000 & 00014 \\
0.0 & \\
\vdots & \\
\vdots & \\
\vdots & \\
0.0 & \end{array}$ & 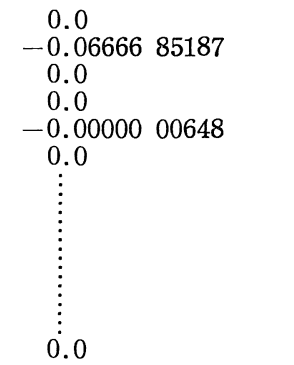 \\
\hline$r$ & $1.15 \times 10^{-8}$ & $2.37 \times 10^{-8}$ & $5 \times 10^{-10}$ \\
\hline$M$ & 70.9 & 71.3 & 16.4 \\
\hline$E$ & $8.155 \times 10^{-7}$ & $1.6902 、 10^{-6}$ & $8.3<10^{-9}$ \\
\hline$\lambda$, & $\begin{array}{l}0.8810883555 \\
\quad \pm 0.4729516979 i \\
\end{array}$ & $\begin{array}{l}1.62112 \quad 277 \mathrm{U}, \\
0.61685 \quad 642 \mathrm{i} 2\end{array}$ & $\begin{array}{l}-0.0006544034 \\
\pm 0.9999997858 i\end{array}$ \\
\hline$\left|\lambda_{l}\right|$ & 0.9999999993 & & 0.9999999999 \\
\hline Stability & neutral & unstable & neutral \\
\hline
\end{tabular}


Table 2.2.1

Periodic solutions to (1.7) with $\sigma=0(\varepsilon=1 / 2, \omega=3.05)$

\begin{tabular}{|c|c|c|c|}
\hline \multirow[b]{2}{*}{$n$} & \multicolumn{2}{|c|}{ Subharmonic solutions } & \multirow{2}{*}{$\frac{\text { Harmonic solution }}{a_{n}}$} \\
\hline & $a_{n}$ & $a_{n}$ & \\
\hline $\begin{array}{c}1 \\
2 \\
3 \\
4 \\
5 \\
6 \\
7 \\
8 \\
9 \\
\vdots \\
\vdots \\
15\end{array}$ & $\begin{array}{rl}0.31340 & 53558 \\
-0.12113 & 75427 \\
-0.00011 & 05583 \\
0.00003 & 47781 \\
-0.00000 & 26472 \\
-0.00000 & 00131 \\
0.00000 & 00015 \\
-0.00000 & 00001 \\
0.0 & \\
\vdots & \\
\vdots & \\
0.0 & \end{array}$ & $\begin{array}{cc}-0.19237 & 42156 \\
-0.12103 & 80304 \\
-0.00011 & 03701 \\
-0.00002 & 13758 \\
-0.00000 & 27122 \\
-0.00000 & 00086 \\
-0.00000 & 00010 \\
-0.00000 & 00001 \\
0.0 & \\
\vdots & \\
\vdots & \\
0.0 & \end{array}$ & $\begin{array}{ll}0.0 & \\
-0.12052 & 47278 \\
0.0 & \\
0.0 & \\
-0.00000 & 26459 \\
0.0 & \\
0.0 & \\
-0.00000 & 00001 \\
0.0 & \\
\vdots \\
\vdots \\
0.0\end{array}$ \\
\hline$r$ & $2.19 \times 10^{-8}$ & $2.05 \times 10^{-8}$ & $1.2 \times 10^{-9}$ \\
\hline$M$ & 60.6 & 97.0 & 9.9 \\
\hline$E$ & $1.3275 \times 10^{-6}$ & $1.9889 \times 10^{-6}$ & $1.19 \times 10^{-8}$ \\
\hline$\lambda_{i}$ & $\begin{array}{l}0.9885125646 \\
\pm 0.1511387076 i\end{array}$ & $\begin{array}{l}1.098806886, \\
0.9100780240\end{array}$ & $\begin{array}{l}-0.4798271512 \\
\pm 0.87736 \quad 30406 i \\
\end{array}$ \\
\hline$\left|\lambda_{i}\right|$ & 0.9999999997 & & 1.000000000 \\
\hline Stability & neutral & unstable & neutral \\
\hline
\end{tabular}

Table 2.2 .2

Periodic solutions to (1.7) with $\sigma=0 \quad(\varepsilon=1 / 2, \omega=3.1)$

\begin{tabular}{|c|c|c|c|}
\hline \multirow[b]{2}{*}{$n$} & \multicolumn{2}{|c|}{ Subharmonic solutions } & \multirow{2}{*}{$\frac{\text { Harmonic solution }}{a_{n}}$} \\
\hline & $a_{n}$ & $a_{n}$ & \\
\hline $\begin{array}{c}1 \\
2 \\
3 \\
4 \\
5 \\
6 \\
7 \\
8 \\
9 \\
\vdots \\
\vdots \\
15\end{array}$ & $\begin{array}{rl}0.45439 & 19264 \\
-0.11695 & 64970 \\
-0.00026 & 33429 \\
0.00004 & 53681 \\
-0.00000 & 22006 \\
-0.00000 & 00253 \\
0.00000 & 00017 \\
0.0 & \\
\vdots \\
\vdots \\
\vdots \\
0.0\end{array}$ & $\begin{array}{cc}-0.33641 & 48660 \\
-0.11793 & 31876 \\
-0.00026 & 41626 \\
-0.00003 & 46282 \\
-0.00000 & 25224 \\
-0.00000 & 00203 \\
-0.00000 & 00015 \\
-0.00000 & 00001 \\
0.0 & \\
\vdots & \\
\vdots & \\
0.0 & \end{array}$ & $\begin{array}{l}0.0 \\
-0.1162123773 \\
0.0 \\
0.0 \\
-0.0000022951 \\
0.0 \\
\vdots \\
\vdots \\
\vdots \\
\vdots \\
0.0\end{array}$ \\
\hline$r$ & $2.34 \times 10^{-8}$ & $2.08 \times 10^{-8}$ & $1.4 \times 10^{-9}$ \\
\hline$M$ & 40.3 & 52.7 & 10.1 \\
\hline$E$ & $9.431 \times 10^{-7}$ & $1.0963 \times 10^{-6}$ & $1.42 \times 10^{-8}$ \\
\hline$\lambda_{i}$ & $\begin{array}{l}0.9663603021 \\
\pm 0.2571920796 i \\
\end{array}$ & $\begin{array}{l}1.21629642, \\
0.8221680007 \\
\end{array}$ & $\begin{array}{r}-0.4495648933 \\
\pm 0.8932476738 i \\
\end{array}$ \\
\hline$\left|\lambda_{\imath}\right|$ & 0.9999999997 & & 1.000000000 \\
\hline Stability & neutral & unstable & neutral \\
\hline
\end{tabular}


Table 2.2.3

Periodic solutions to (1.7) with $\sigma=0 \quad(\varepsilon=1 / 2, \omega=3.2)$

\begin{tabular}{|c|c|c|c|}
\hline \multirow[b]{2}{*}{$n$} & \multicolumn{2}{|c|}{ Subharmonic solutions } & \multirow{2}{*}{$\frac{\text { Harmonic solution }}{a_{n}}$} \\
\hline & $a_{n}$ & $a_{n}$ & \\
\hline $\begin{array}{c}1 \\
2 \\
3 \\
4 \\
5 \\
6 \\
7 \\
8 \\
9 \\
\vdots \\
\vdots \\
15\end{array}$ & $\begin{array}{rr}0.64303 & 26531 \\
-0.10832 & 12797 \\
-0.00051 & 46997 \\
0.00005 & 10049 \\
-0.00000 & 13945 \\
-0.00000 & 00368 \\
0.00000 & 00014 \\
0.0 & \\
\vdots & \\
\vdots & \\
\vdots & \\
0.0 & \end{array}$ & $\begin{array}{cc}-0.53088 & 90496 \\
-0.11289 & 93867 \\
-0.00053 & 17972 \\
-0.00004 & 80110 \\
-0.00000 & 23212 \\
-0.00000 & 00375 \\
-0.00000 & 00019 \\
-0.00000 & 00001 \\
0.0 & \\
\vdots & \\
\vdots & \\
0.0 & \end{array}$ & $\begin{array}{ll}0.0 & \\
-0.10827 & 66276 \\
0.0 & \\
0.0 & \\
-0.00000 & 17408 \\
0.0 & \\
\vdots \\
\vdots \\
\vdots \\
\vdots \\
\vdots \\
0.0\end{array}$ \\
\hline$r$ & $1.21 \times 10^{-8}$ & $1.59 \times 10^{-8}$ & $1.0 \times 10^{-9}$ \\
\hline$M$ & 31.5 & 36.4 & 10.7 \\
\hline$E$ & $3.812 \times 10^{-7}$ & $5.788 \times 10^{-7}$ & $1.08 \times 10^{-8}$ \\
\hline$\lambda_{i}$ & $\begin{array}{l}0.9196876243 \\
\pm 0.3926508284 i\end{array}$ & $\begin{array}{l}1.405032563 \\
0.7117272764 \\
\end{array}$ & $\begin{array}{l}-0.3906279056 \\
\quad \pm 0.9205486621 i\end{array}$ \\
\hline$\left|\lambda_{i}\right|$ & 0.9999999997 & & 0.9999999999 \\
\hline Stability & neutral & unstable & neutral \\
\hline
\end{tabular}

Table 2.2.4

Periodic solutions to (1.7) with $\sigma=0 \quad(\varepsilon=1 / 2, \omega=4)$

\begin{tabular}{|c|c|c|c|}
\hline \multirow[b]{2}{*}{$n$} & \multicolumn{2}{|c|}{ Subharmonic solutions } & \multirow{2}{*}{$\frac{\text { Harmonic solution }}{a_{n}}$} \\
\hline & $a_{n}$ & $a_{n}$ & \\
\hline $\begin{array}{c}1 \\
2 \\
3 \\
4 \\
5 \\
6 \\
7 \\
8 \\
9 \\
\vdots \\
\vdots \\
15\end{array}$ & $\begin{array}{rr}1.46165 & 38180 \\
-0.04555 & 49212 \\
-0.00084 & 49916 \\
0.00000 & 59508 \\
0.00000 & 02492 \\
-0.00000 & 00018 \\
-0.00000 & 00001 \\
0.0 & \\
\vdots & \\
\vdots & \\
\vdots & \\
0.0 & \end{array}$ & $\begin{array}{cc}-1.38496 & 30370 \\
-0.09836 & 10693 \\
-0.00180 & 57494 \\
-0.00007 & 68957 \\
-0.00000 & 26025 \\
-0.00000 & 00865 \\
-0.00000 & 00030 \\
-0.00000 & 00001 \\
0.0 & \\
\vdots & \\
\vdots & \\
0.0 & \end{array}$ & $\begin{array}{ll} & 0.0 \\
-0.06667 & 40766 \\
0.0 & \\
0.0 & \\
-0.00000 & 02591 \\
0.0 & \\
\vdots \\
\vdots \\
\vdots \\
\vdots \\
0.0\end{array}$ \\
\hline$r$ & $7.0 \times 10^{-9}$ & $1.49 \times 10^{-8}$ & $2 \times 10^{-10}$ \\
\hline$M$ & 35.6 & 35.9 & 16.3 \\
\hline$E$ & $2.493 \times 10^{-7}$ & $5.350 \times 10^{-7}$ & $3.3 \times 10^{-9}$ \\
\hline$\lambda_{i}$ & $\begin{array}{l}0.7635740830 \\
\quad \pm 0.64572 \quad 02327 i \\
\end{array}$ & $\begin{array}{l}1.964148870 \\
0.5091263771\end{array}$ & $\begin{array}{c}-0.0026164669 \\
\pm 0.9999965770 i\end{array}$ \\
\hline$\left|\lambda_{1}\right|$ & 0.9999999995 & & 0.9999999999 \\
\hline Stability & neutral & unstable & neutral \\
\hline
\end{tabular}


Table 2.3.1

Periodic solutions to (1.7) with $\sigma=0 \quad(\varepsilon=1, \omega=3.05)$

\begin{tabular}{|c|c|c|c|}
\hline \multirow[b]{2}{*}{$n$} & \multicolumn{2}{|c|}{ Subharmonic solutions } & \multirow{2}{*}{$\frac{\text { Harmonic solution }}{a_{n}}$} \\
\hline & $a_{n}$ & $a_{n}$ & \\
\hline $\begin{array}{c}1 \\
2 \\
3 \\
4 \\
5 \\
6 \\
7 \\
8 \\
9 \\
\vdots \\
\vdots \\
15\end{array}$ & $\begin{array}{rl}0.19874 & 95570 \\
-0.12123 & 55889 \\
-0.00005 & 65814 \\
0.00004 & 42107 \\
-0.00000 & 53693 \\
-0.00000 & 00176 \\
0.00000 & 00039 \\
-0.00000 & 00002 \\
0.0 & \\
\vdots & \\
\vdots & \\
0.0 & \end{array}$ & $\begin{array}{cc}-0.07843 & 84541 \\
-0.12075 & 36311 \\
-0.00005 & 70524 \\
-0.00001 & 73127 \\
-0.00000 & 53370 \\
-0.00000 & 00078 \\
-0.00000 & 00015 \\
-0.00000 & 00003 \\
0.0 & \\
\vdots & \\
\vdots & \\
0.0 & \end{array}$ & $\begin{array}{ll}0.0 & \\
-0.12060 & 41229 \\
0.0 & \\
0.0 & \\
-0.00000 & 53029 \\
0.0 & \\
0.0 & \\
-0.00000 & 00002 \\
0.0 & \\
\vdots \\
\vdots \\
0.0\end{array}$ \\
\hline$r$ & $2.46 \times 10^{-8}$ & $2.47 \times 10^{-8}$ & $1.8 \times 10^{-9}$ \\
\hline$M$ & 63.9 & 158.8 & 9.8 \\
\hline$E$ & $1.5723 \times 10^{-6}$ & $3.9256 \times 10^{-6}$ & $1.77 \times 10^{-8}$ \\
\hline$\lambda_{i}$ & $\begin{array}{l}0.9898412174 \\
\quad \pm 0.14217 \quad 72262 i\end{array}$ & $\begin{array}{l}1.058690072, \\
0.9445634995\end{array}$ & $\begin{array}{l}-0.4895937021 \\
\pm 0.87195 \quad 06906 i\end{array}$ \\
\hline$\left|\lambda_{2}\right|$ & 0.9999999997 & & 1.000000000 \\
\hline Stability & neutral & unstable & neutral \\
\hline
\end{tabular}

Table 2.3.2

Periodic solutions to (1.7) with $\sigma=0 \quad(\varepsilon=1, \omega=3.1)$

\begin{tabular}{|c|c|c|c|}
\hline \multirow[b]{2}{*}{$n$} & \multicolumn{2}{|c|}{ Subharmonic solutions } & \multirow{2}{*}{$\frac{\text { Harmonic solution }}{a_{n}}$} \\
\hline & $a_{n}$ & $a_{n}$ & \\
\hline $\begin{array}{c}1 \\
2 \\
3 \\
4 \\
5 \\
6 \\
7 \\
8 \\
9 \\
\vdots \\
\vdots \\
15\end{array}$ & $\begin{array}{rl}0.31600 & 21647 \\
-0.11741 & 22723 \\
-0.00021 & 64496 \\
0.00006 & 37913 \\
-0.00000 & 45871 \\
-0.00000 & 00459 \\
0.00000 & 00050 \\
-0.00000 & 00002 \\
0.0 & \\
\vdots & \\
\vdots & \\
0.0 & \end{array}$ & $\begin{array}{cc}-0.19866 & 15617 \\
-0.11732 & 07872 \\
-0.00021 & 57463 \\
-0.00004 & 03006 \\
-0.00000 & 48456 \\
-0.00000 & 00309 \\
-0.00000 & 00033 \\
-0.00000 & 00002 \\
0.0 & \\
\vdots & \\
\vdots & \\
0.0 & \end{array}$ & $\begin{array}{ll}0.0 & \\
-0.11628 & 09810 \\
0.0 & \\
0.0 & \\
-0.00000 & 45989 \\
0.0 & \\
0.0 & \\
-0.00000 & 00002 \\
0.0 & \\
\vdots & \\
\vdots & \\
0.0 & \end{array}$ \\
\hline$r$ & $2.60 \times 10^{-8}$ & $1.35 \times 10^{-8}$ & $3 \times 10^{-10}$ \\
\hline$M$ & 32.0 & 49.3 & 10.1 \\
\hline$E$ & $8.321 \times 10^{-7}$ & $6.656 \times 10^{-7}$ & $3.1 \times 10^{-9}$ \\
\hline$\therefore$ & $\begin{array}{l}0.9577642812 \\
\quad \pm 0.28755 \quad 44838 i \\
\end{array}$ & $\begin{array}{l}1.206104705 \\
0.8291154117 \\
\end{array}$ & $\begin{array}{r}-0.45866 \quad 69814 \\
\pm 0.88860 \quad 82377 i \\
\end{array}$ \\
\hline$|\lambda|$, & 0.9999999998 & & 1.000000000 \\
\hline Stability & neutral & unstable & neutral \\
\hline
\end{tabular}


Table 2.3.3

Periodic solutions to (1.7) with $\sigma=0 \quad(\varepsilon=1, \omega=3.2)$

\begin{tabular}{|c|c|c|c|}
\hline \multirow[b]{2}{*}{$n$} & \multicolumn{2}{|c|}{ Subharmonic solutions } & \multirow{2}{*}{$\frac{\text { Harmonic solution }}{a_{n}}$} \\
\hline & $a_{n}$ & $a_{n}$ & \\
\hline $\begin{array}{c}1 \\
2 \\
3 \\
4 \\
5 \\
6 \\
7 \\
8 \\
9 \\
\vdots \\
\vdots \\
15\end{array}$ & $\begin{array}{rr}0.45801 & 73216 \\
-0.10946 & 46843 \\
-0.00048 & 31870 \\
0.00007 & 49046 \\
-0.00000 & 31416 \\
-0.00000 & 00747 \\
0.00000 & 00045 \\
-0.00000 & 00001 \\
0.0 & \\
\vdots & \\
\vdots & \\
0.0 & \end{array}$ & $\begin{array}{cc}-0.34637 & 31608 \\
-0.11164 & 50565 \\
-0.00048 & 78993 \\
-0.00006 & 06922 \\
-0.00000 & 42355 \\
-0.00000 & 00644 \\
-0.00000 & 00045 \\
-0.00000 & 00002 \\
0.0 & \\
\vdots & \\
\vdots & \\
0.0 & \end{array}$ & $\begin{array}{lll} & 0.0 \\
-0.10832 & 82962 \\
& 0.0 \\
& 0.0 & \\
-0.00000 & 34869 \\
& 0.0 & \\
& 0.0 & \\
-0.00000 & 00001 \\
0.0 & \\
\vdots & \vdots \\
\vdots & & \\
0.0 & \end{array}$ \\
\hline$r$ & $2.48 \times 10^{-8}$ & $9.9 \times 10^{-9}$ & $1.1 \times 10^{-9}$ \\
\hline$M$ & 23.2 & 28.8 & 10.7 \\
\hline$E$ & $5.754 \times 10^{-7}$ & $2.852 \times 10^{-7}$ & $1.18 \times 10^{-8}$ \\
\hline$\lambda_{i}$ & $\begin{array}{l}0.8893986110 \\
\pm 0.45713 \quad 24868 i\end{array}$ & $\begin{array}{l}1.44677 \quad 1135 \\
0.69119 \\
43257\end{array}$ & $\begin{array}{l}-0.3985258925 \\
\quad \pm 0.9171570819 i\end{array}$ \\
\hline$\left|\lambda_{i}\right|$ & 0.9999999998 & & 0.9999999999 \\
\hline Stability & neutral & unstable & neutral \\
\hline
\end{tabular}

Table 2.3.4

Periodic solutions to (1.7) with $\sigma=0 \quad(\varepsilon=1, \omega=4)$

\begin{tabular}{|c|c|c|c|}
\hline \multirow[b]{2}{*}{$n$} & \multicolumn{2}{|c|}{ Subharmonic solutions } & \multirow{2}{*}{$\frac{\text { Harmonic solution }}{a_{n}}$} \\
\hline & $a_{n}$ & $a_{n}$ & \\
\hline $\begin{array}{c}1 \\
2 \\
3 \\
4 \\
5 \\
6 \\
7 \\
8 \\
9 \\
\vdots \\
\vdots \\
15\end{array}$ & $\begin{array}{rr}1.04265 & 32218 \\
-0.05366 & 54105 \\
-0.00099 & 23715 \\
0.00001 & 80707 \\
0.00000 & 04148 \\
-0.00000 & 00123 \\
-0.00000 & 00002 \\
0.0 & \\
\vdots & \\
\vdots & \\
\vdots & \end{array}$ & $\begin{array}{cc}-0.96604 & 99115 \\
-0.09024 & 34248 \\
-0.00164 & 48577 \\
-0.00008 & 58323 \\
-0.00000 & 33351 \\
-0.00000 & 01232 \\
-0.00000 & 00049 \\
-0.00000 & 00002 \\
0.0 & \\
\vdots & \\
\vdots & \end{array}$ & $\begin{array}{ll} & 0.0 \\
-0.06668 & 14915 \\
0.0 & \\
0.0 & \\
-0.00000 & 05184 \\
0.0 & \\
\vdots \\
\vdots \\
\vdots \\
\vdots \\
0.0\end{array}$ \\
\hline$r$ & $1.55 \times 10^{-8}$ & $1.48 \times 10^{-8}$ & $5 \times 10^{-10}$ \\
\hline$M$ & 25.3 & 25.7 & 16.3 \\
\hline$E$ & $3.922 \times 10^{-7}$ & $3.804 \times 10^{-7}$ & $8.2 \times 10^{-9}$ \\
\hline$\lambda_{i}$ & $\begin{array}{l}0.6681427831 \\
\quad \pm 0.7440330775 i \\
\end{array}$ & $\begin{array}{l}2.211941080 \\
0.4520916072 \\
\end{array}$ & $\begin{array}{r}-0.0052298781 \\
\pm 0.9999863240 i \\
\end{array}$ \\
\hline$\left|\lambda_{2}\right|$ & 0.9999999995 & & 0.9999999999 \\
\hline Stability & neutral & unstable & neutral \\
\hline
\end{tabular}




\section{Table 3.1.1}

Periodic solutions to (1.7) with $\sigma=2^{-10}(\varepsilon=1 / 8, \omega=3.1)$

$1^{\circ} \quad \bar{x}_{15}(t)=0.0302880058 \sin t \quad+0.8933182959 \cos t$ $+0.0003091923 \sin 3 t-0.1155953736 \cos 3 t$ $-0.0000237202 \sin 5 t-0.0002944095 \cos 5 t$ $+0.0000005627 \sin 7 t+0.0000215577 \cos 7 t$ $+0.0000000139 \sin 9 t-0.0000004849 \cos 9 t$ $-0.0000000004 \sin 11 t-0.0000000063 \cos 11 t$ $+0.0000000002 \cos 13 t$, $r=1.9 \times 10^{-9}, M=76.6, E=1.456 \times 10^{-7}, \delta_{0}=1.47 \times 10^{-7}$, $\lambda_{1}, \lambda_{2}=0.9798803088 \pm 0.1841580459 i$,

$\left|\lambda_{1}\right|, \quad\left|\lambda_{2}\right|=0.9970354082$, Stability : stable.

$2^{\circ} \quad \bar{x}_{15}(t)=0.0306335257 \sin t \quad-0.7754834440 \cos t$ $+0.0002457725 \sin 3 t-0.1194161668 \cos 3 t$ $+0.0000199608 \sin 5 t-0.0003033481 \cos 5 t$ $+0.0000009372 \sin 7 t-0.0000206791 \cos 7 t$ $+0.0000000123 \sin 9 t-0.0000007027 \cos 9 t$ $+0.0000000005 \sin 11 t-0.0000000064 \cos 11 t$ $-0.0000000002 \cos 13 t$, $r=2.0 \times 10^{-9}, M=86.5, E=1.731 \times 10^{-7}, \delta_{0}=1.8 \times 10^{-7}$, $\lambda_{1}=1.173264292, \lambda_{2}=0.8472767891$,

Stability: unstable.

\section{Table 3.1.2}

Periodic solutions to (1.7) with $\sigma=2^{-10}(\varepsilon=1 / 8, \omega=4)$

$1^{\circ} \quad \bar{x}_{15}(t)=\quad+0.0000000001$

$+0.0621004049 \sin t+2.8886467924 \cos t$

$-0.0000000001 \sin 2 t$

$+0.0036247077 \sin 3 t-0.0184637771 \cos 3 t$

$+0.0000519900 \sin 5 t-0.0003455740 \cos 5 t$

$-0.0000000777 \sin 7 t-0.0000021181 \cos 7 t$

$-0.0000000082 \sin 9 t+0.0000000113 \cos 9 t$

$-0.0000000001 \sin 11 t+0.0000000002 \cos 11 t$, $r=4.1 \times 10^{-9}, M=71.0, E=2.912 \times 10^{-7}, \delta_{0}=3 \times 10^{-7}$, $\lambda_{1}, \lambda_{2}=0.8793224423 \pm 0.4713816650 i$, $\left|\lambda_{1}\right|=\left|\lambda_{2}\right|=0.9977016747$, Stability : stable.

$2^{\circ}$

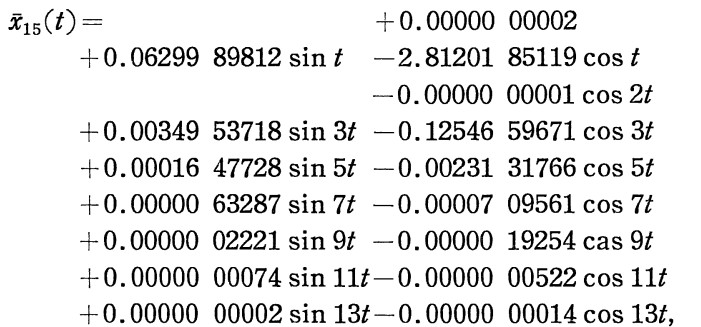
$r=4.5 \times 10^{-9}, M=71.4, E=3.214 \times 10^{-7}, \delta_{0}=3.3 \times 10^{-7}$, $\lambda_{1}=1.616576060, \lambda_{2}=0.6157511731$,

Stability : unstable. 
Table 3.2.1

Periodic solutions to (1.7) with $\sigma=2^{-10} \quad(\varepsilon=1, \omega=3.1)$

$1^{\circ} \quad \bar{x}_{15}(t)=0.0037484022 \sin t+0.3159324676 \cos t$

$+0.0000753137 \sin 3 t-0.1174126343 \cos 3 t$

$-0.0000095962 \sin 5 t-0.0002162865 \cos 5 t$

$+0.0000006531 \sin 7 t+0.0000637792 \cos 7 t$

$+0.0000000195 \sin 9 t-0.0000045873 \cos 9 t$

$-0.0000000014 \sin 11 t-0.0000000458 \cos 11 t$

$+0.0000000050 \cos 13 t$

$-0.0000000002 \cos 15 t$,

$r=2.9 \times 10^{-9}, M=32.1, E=9.31 \times 10^{-8}, \delta_{0}=1 \times 10^{-7}$, $\lambda_{1}, \lambda_{2}=0.9549822496 \pm 0.2865109291 i$,

$\left|\lambda_{1}\right|=\left|\lambda_{2}\right|=0.9970354104$,

Stability : stable.

$2^{\circ} \quad \bar{x}_{15}(t)=0.0038511023 \sin t \quad-0.1986989516 \cos t$

$+0.0000549550 \sin 3 t-0.1173212521 \cos 3 t$

$+0.0000038874 \sin 5 t-0.0002157613 \cos 5 t$

$+0.0000008258 \sin 7 t-0.0000403077 \cos 7 t$

$+0.0000000114 \sin 9 t-0.0000048456 \cos 9 t$

$+0.0000000008 \sin 11 t-0.0000000309 \cos 11 t$

$+0.0000000001 \sin 13 t-0.0000000033 \cos 13 t$

$-0.0000000002 \cos 15 t$,

$r=2.2 \times 10^{-9}, M=49.3, E=1.085 \times 10^{-7}, \delta_{0}=1.1 \times 10^{-7}$,

$\lambda_{1}=1.202528159, \lambda_{2}=0.8266580649$,

Stability: unstable.

Table 3.2.2

Periodic solutions to (1.7) with $\sigma=2^{-10}(\varepsilon=1, \omega=4)$

$1^{\circ} \quad \bar{x}_{15}(t)=0.0076538598 \sin t+1.0426149858 \cos t$

$+0.0004830974 \sin 3 t-0.0536709094 \cos 3 t$

$-0.0000071715 \sin 5 t-0.0009924868 \cos 5 t$

$-0.0000005051 \sin 7 t+0.0000180779 \cos 7 t$

$+0.0000000098 \sin 9 t+0.0000004150 \cos 9 t$

$+0.0000000005 \sin 11 t-0.0000000123 \cos 11 t$

$-0.0000000002 \cos 13 t$,

$r=1.6 \times 10^{-9}, M=25.3, \quad E=4.05 \times 10^{-8}, \delta_{0}=4.2 \times 10^{-8}$,

$\lambda_{1}, \lambda_{2}=0.6666989022 \pm 0.7422406603 i$,

$\left|\lambda_{1}\right|=\left|\lambda_{2}\right|=0.9977016708$,

Stability : stable.

$2^{\circ} \quad \bar{x}_{15}(t)=$

$+0.0000000001$

$+0.0079697109 \sin t \quad-0.9660278183 \cos t$

$+0.0004369056 \sin 3 t-0.0902389741 \cos 3 t$

$+0.0000325368 \sin 5 t-0.0016444793 \cos 5 t$

$+0.0000017935 \sin 7 t-0.0000858069 \cos 7 t$

$+0.0000000868 \sin 9 t-0.0000033336 \cos 9 t$

$+0.0000000042 \sin 11 t-0.0000001231 \cos 11 t$

$+0.0000000002 \sin 13 t-0.0000000049 \cos 13 t$

$-0.0000000002 \cos 15 t$,

$r=3.9 \times 10^{-9}, M=25.7, E=1.003 \times 10^{-7}, \delta_{0}=1.08 \times 10^{-7}$, $\lambda_{1}=2.206639824, \lambda_{2}=0.4510970035$,

Stability : unstable. 


\section{Table 4}

Periodic solutions to (1.3) with $\sigma=2^{-4}$.

$1^{\circ} \varepsilon=1 / 8, \omega=3.1$

$\bar{x}_{15}(t)=0.0026130219 \sin t \quad-0.1161022676 \cos t$

$+0.0000000425 \sin 3 t-0.0000005709 \cos 3 t$, $r=2 \times 10^{-10}, M=10.2, \quad E=2.1 \times 10^{-9}, \delta_{0}=2.1 \times 10^{-9}$,

$\lambda_{1}, \lambda_{2}=-0.4146898606 \pm 0.8420513087 i$,

$\left|\lambda_{1}\right|=\left|\lambda_{2}\right|=0.9386256373$,

Stability : stable

$2^{\circ} \varepsilon=1 / 8, \omega=4.0$.

$\bar{x}_{15}(t)=0.0011108642 \sin t \quad-0.0666500033 \cos t$

$+0.0000000036 \sin 3 t-0.0000000646 \cos 3 t$,

$r=1 \times 10^{-10}, M=16.4, E=1.7 \times 10^{-9}, \delta_{0}=1.9 \times 10^{-9}$,

$\lambda_{1}, \lambda_{2}=0.0001072410 \pm 0.9520979207 i$,

$\left|\lambda_{1}\right|=\left|\lambda_{2}\right|=0.9520979267$,

Stability : stable.

$3^{\circ} \varepsilon=1, \omega=3.1$.

$\bar{x}_{15}(t)=0.0026184132 \sin t \quad-0.1162219199 \cos t$

$+0.0000003415 \sin 3 t-0.0000045826 \cos 3 t$

$-0.0000000002 \cos 5 t$,

$r=2 \times 10^{-10}, M=10.1, E=2.1 \times 10^{-9}, \delta_{0}=2.7 \times 10^{-9}$,

$\lambda_{1}, \lambda_{2}=-0.4296903383 \pm 0.8344964351 i$,

$\left|\lambda_{1}\right|=\left|\lambda_{2}\right|=0.9386256373$,

Stability : stable.

$4^{\circ} \quad \varepsilon=1, \omega=4$.

$\bar{x}_{15}(t)=0.0011112964 \sin t \quad-0.0666629616 \cos t$

$+0.0000000286 \sin 3 t-0.0000005174 \cos 3 t$,

$r=1 \times 10^{-10}, M=16.3, E=1.7 \times 10^{-9}, \delta_{0}=2 \times 10^{-9}$,

$\lambda_{1}, \lambda_{2}=-0.0042499789 \pm 0.9520884411 i$,

$\left|\lambda_{1}\right|=\left|\lambda_{2}\right|=0.9520979267$,

Stability : stable.

\section{References}

[1] Cesari, L., Functional analysis and periodic solutions of nonlinear differential equations, Contributions to Differential Equations, Vol. 1, No. 2, pp. 149-187, Wiley, New York, 1963.

[2] Christopher, P. A.T., Exact subharmonic oscillations, College of Aeronautics, Cranfield, Report Aero. 190 (1965).

[3] ——, A new class of subharmonic solutions to Duffing's equation, Ibid. 195 (1967).

[4] - An extended class of subharmonic solutions to Duffing's equation, Ibid. 199 (1967).

[5] Hayashi, C., Nonlinear oscillations in physical systems, McGraw-Hill, New York, 1964.

[6] Kauderer, H., Nichtlineare Mechanik, Springer, Berlin, 1958. 
[7] Levenson. M.E., A numerical determination of subharmonic response for the Duffing equation $\ddot{x}+\alpha x+\beta x^{3}=F \cos \omega t(\alpha>0)$, Quart. Appl. Math. 25 (1967), 11-17.

[8] - A numerical determination of ultra-subharmonic response for the Duffing equation $\ddot{x}+\alpha x+\beta x^{3}=F \cos \omega t(\alpha>0)$, Ibid. 26 (1968), 456-461.

[9] Ludeke, C. A. and J. E. Cornett, A computer investigation of a subharmonic bifurcation point in the Duffing equation, SIAM J. Appl. Math. 14 (1966), 1298-1306.

[10] Малкин, И. Г., Некоторые эадачп теорпп нелинеиных колебаншп, Гос. Изд., Москва, 1956.

[11] Minorsky, N., Nonlinear oscillations, Van Nostrand, Princeton, 1962.

[12] Stoker, J. J., Nonlinear vibrations in mechanical and electrical systems, Interscience, New York, 1950.

[13] Urabe, M., Galerkin's procedure for nonlinear periodic systems, Arch. Rational Mech. Anal. 20 (1965), 120-152.

[14] - Numerical solution of multi-point boundary value problems in Chebyshev series - theory of the method, Numer. Math. 9 (1967), 341-366.

[15] - Numerical solution of boundary value problems in Chebyshev seriesa method of computation and error estimation, to appear.

[16] Urabe, M. and A. Reiter, Numerical computation of nonlinear forced oscillations by Galerkin's procedure, J. Math. Anal. Appl. 14 (1966), 107-140. 